\title{
Exosomal miRNA-21-5p derived from bone marrow mesenchymal stem cells promote osteosarcoma cell proliferation and invasion by targeting PIK3R1
}

Jin Qi (D815018560@qq.com )

Lanzhou University Second Hospital

Ruihao Zhang

Lanzhou University Second Hospital

Zuolong Wu

Lanzhou University Second Hospital

Yayi Xia

Lanzhou University Second Hospital

\section{Research Article}

Keywords: exosomes, miR-21-5p, MSCs, Osteosarcoma, PIK3R1, PI3K/Akt/mTOR pathway

Posted Date: December 10th, 2020

DOI: https://doi.org/10.21203/rs.3.rs-120370/v1

License: (9) This work is licensed under a Creative Commons Attribution 4.0 International License.

Read Full License 


\title{
Exosomal miRNA-21-5p derived from bone marrow mesenchymal stem cells promote osteosarcoma cell proliferation and invasion by targeting PIK3R1
}

\author{
Jin $\mathrm{Qi}^{1,2^{*}}$, Ruihao Zhang ${ }^{2}$, Zuolong $\mathrm{Wu}^{2}$, Yayi Xia ${ }^{12}$
}

1 Lanzhou University Second hospital, Lanzhou, People's Republic of China. 2 Orthopaedics Key Laboratory of Gansu Province, Lanzhou, People's Republic of China. Correspondence to email:815018560@qq.com

Abstract Mesenchymal stem cells (MSCs) are a class of pluripotent cells that can release a large number of exosomes which act as paracrine mediators in tumor associated microenvironment. However, the role of MSC-derived exosomes in pathogenesis and progression of cancer cells especially osteosarcoma has not yet been thoroughly clarified until now. In this study, we established a co-culture model for human bone marrow derived MSCs with osteosarcoma U2OS and MG63 cells, then extraction of exosomes from induced MSCs and study the role of MSC-derived exosomes in the progression of osteosarcoma cell. It was the aim of this study to address potential cell biological effects between MSCs and osteosarcoma cell. we found that MSC-derived exosomes can significantly promote osteosarcoma cells proliferation and invasion. we also found that miR-21-5p were significantly overexpressed in human bone marrow MSCs and MSC-derived exosomes compared with that of human fetal osteoblastic cell line hFOB1.19 by using quantitative realtime polymerase chain reaction (qRT-PCR). Proliferation and invasion of osteosarcoma cells U2OS and MG63 were significantly enhanced by MSC-derived exosomes that were transfected with miR-21-5p. Bioinformatics analysis and duatluciferase reporter gene assays validated the targeted relationship between exosomal miR-21-5p and PIK3R1. Furthermore, we demonstrated that miR-21-5p-abundant exosomes derived human bone marrow MSCs could activate PI3K/Akt/mTOR pathway by suppressing PIK3R1 expression in osteosarcoma cells U2OS and MG63. In conclusion, Our findings provide new insight into the interaction between MSCs and osteosarcoma cells in tumor associated microenvironment. Notably, the use of a miR-21-5p inhibitor has an excellent restraining effect on osteosarcoma proliferation and invasion, which provides therapeutic potential for osteosarcoma in future clinical medicine.

Keywords: exosomes, miR-21-5p, MSCs, Osteosarcoma, PIK3R1, PI3K/Akt/mTOR pathway

\section{Introduction}

Osteosarcoma (OS) is the most common primary malignant bone cancer in children and adolescents. Recurrence and metastasis are the main reasons for the unsatisfactory treatment of OS[1]. However, the mechanism of proliferation and invasion of OS is still not clear.

As we all know, the biological behavior of tumor is not entirely determined by tumor cells alone, and stromal cell in tumor associated microenvironment act on tumor cells to promote tumor proliferation and invasion, which has become more and more concerned. As a non-tumor cell, Mesenchymal stem cells (MSCs) may participate in the processes of OS proliferation and invasion 
in OS associated microenvironment[2]. MSCs are a kind of non-hematopoietic progenitor cells with multipotent and self-renewable abilities that reside in many tissues such as bone marrow, umbilical cord, adipose tissue, and placenta tissue, where they are involved in maintenance and regeneration of surrounded tissues[3-6]. Because MSCs have biological characteristics of migration to cancer site where they participate in the formation of tumor associated microenvironment, MSCs have always been studied in the field of cancer pathogenesis and cancer therapy[7, 8]. MSCs not only provide the microenvironment for cancer cells, but also enhance cancer progression and invasion[9]. It has been found that the aneuploidy and genomic loss can transform MSCs into OS cells, indicating that OS cells may originate from bone marrow MSCs[10]. Studying the role of MSCs in OS proliferation and invasion is of great significance to elucidate the mechanism of OS progression.

Classically, MSCs play its role mainly through cell contact-dependent mechanisms and soluble factors[11]. However, more and more studies have found that MSCs could release large amounts of exosomes through paracrine mechanism, which can change the microenvironment around target cells, thus regulating the biological functions of cell proliferation, differentiation, apoptosis[12-14]. Exosomes carry a variety of types of RNA, DNA, protein and other signal molecules of donor cells[15]. Its main biological function is to transmit the signal molecules to recipient cells, thus forming the information exchange and transmission between cells[16]. Exosomes participate in the pathological process of various diseases, including cancer[17].

In previous studies, we found that MSC-exosomes enhance the proliferation of OS cells, but we have not yet elucidated the specific molecular biological mechanism. Recently, it has been found that the expression of miRNA-21-5p increased during the osteogenic differentiation of MSCs[18]. MiRNA-21-5p is recognized as an oncogene that can promote the proliferation and invasion of cancer[19]. Here, we found MSC-exosomes may possess abundant miR-21-5p to further influence the biological properties of OS, and the exosomes protect RNA-21-5p from RNase degradation in OS associated microenvironment. Bioinformatics prediction found that PIK3R1 is the target gene of miR-21-5p and is also responsible for encoding an important regulatory subunit (p85 $\alpha$ ) of Phosphatidylinositol 3-Kinases(PI3K), PI3K form heterodimeric protein complexes composed of a catalytic p110 subunit encoded by the PIK3CA and a p85 regulatory subunit[20, 21]. The PIK3CA oncogene carrying a hotspot mutation exerts an oncogenic activity, on the contrary, the PIK3R1 appears to play a tumor suppressor role because PI3K subunit $\mathrm{p} 85 \alpha$ regulates and stabilizes p110[21-23].P85-type also have other two subunits: PIK3R2 and PIK3R3. PIK3R1 and PIK3R2 are broadly expressed in tissues, whereas PIK3R3 is selectively expressed in adult testis and the brain[24]. Classically, $\mathrm{p} 85 \mathrm{a}$ and $\mathrm{p} 85 \mathrm{~b}$ have been considered to be similar proteins that associate with activated receptor tyrosine kinases (RTK) that induce PI3K activation, however, PIK3R1(p85 $\alpha$ ) is the most abundant isoform in normal tissues but its expression is reduced in cancer, which has a tumor-suppressor function[24]. Conversely, PIK3R2/p85 $\beta$ expression levels are elevated in cancer which is regarded as a tumor driver[25-27]. Physiological activation of $\mathrm{PI} 3 \mathrm{~K}$ is induced by binding of $\mathrm{p} 85$ to activated receptor tyrosine kinases (RTKs)[28].

$\mathrm{PI} 3 \mathrm{~K} / \mathrm{Akt} / \mathrm{mTOR}$ is one of the signaling pathways closely related to apoptosis and cell proliferation, which is usually highly activated in OS[29].In this study, we hypothesized that miR-21-5p-abundant MSC-exosomes might participate in OS cell proliferation and invasion through activated PI3K/Akt/mTOR signaling pathway, and miR-21-5p expression in MSC-exosomes may serve as a therapeutic target for OS in the future. 


\section{Materials and Methods}

\section{Cell culture}

Human U2OS and MG63 cell line (purchased from Cell Bank, Chi Scientific, Inc) were cultured in DMEM with $10 \%$ fetal bovine serum, $1 \%$ penicillin-streptomycin. All cells cultured in an incubator with a humidify atmosphere of $5 \% \mathrm{CO}_{2}$ at $37^{\circ} \mathrm{C}$. Primary human MSCs were isolated from bone marrow of the patient who have been diagnosed as OS and were adhere-wall cultured to isolation, it has been detailedly described as our previous reported[30]. All patients agreed voluntarily to participate in the study which was approved by the ethics committee of Lanzhou university second hospital (Lanzhou, china). Isolated MSCs were co-cultured with OS, and induced MSCs were culutred in complete DMEM/F12 supplemented with 10\% exosome-depleted fetal bovine serum and $1 \%$ penicillin-streptomycin. When wall-adhered MSCs reached $80 \%$ confluence, culture supernatants from MSCs was harvested. The 3rd passage of MSCs was collected for in-vitro phenotype analysis.

\section{Identification of MSCs and MSC-derived exosomes}

Cellular surface antigens of MSCs were examined with flow-cytometry and it has been detailedly described in previous experiment[31]. The osteogenic and adipogenic differentiation of MSCs were further performed by using the differentiation media and detected by Oil red $\mathrm{O}$ staining and Alizarin red staining(Cyagen Bioscience,Inc.). Cellular surface antigens were examined with flow-cytometry of MSCs for CD19, CD29, CD90, CD44, CD73, CD105 and CD133 markers,

MSC-exosomes were purified according to the exosome extraction protocol[32]. In brief, cell culture supernatants were centrifuged at $300 \times \mathrm{g}$ for $10 \mathrm{~min}$ to remove cells, $2000 \times \mathrm{g}$ for $10 \mathrm{~min}$ to remove dead cells, and then $10,000 \times \mathrm{g}$ for $30 \mathrm{~min}$ to remove cell debris, and the supernatant was kept for the next step ultracentrifugation. After the two 100, $000 \times \mathrm{g}$ ultracentrifugations for 70min, pellets (exosomes) are kept, and supernatants are discarded. All centrifugations should be performed at $4^{\circ} \mathrm{C}$. The aliquots were passed through $0.22-\mu \mathrm{m}$ filters, the pellet was carefully resuspended in PBS and used immediately or stored at $-80^{\circ} \mathrm{C}$. Purified exosomes were negatively stained with uranyl acetate by means of floating method, and observed by transmission electron microscopy.

\section{Co-culture experiments}

MSCs were inoculated at 6-well plate with a density of $1 \times 10^{4} \mathrm{cells} / \mathrm{cm}^{2}$, and OS cells seeded in 6 -well chambers with $0.4 \mu \mathrm{m}$ polycarbonate membrane pores. The membrane was permeable, and cells could not pass through the membrane, but the cytokines secreted by cells could pass through. Cells were co-culture for 2 weeks, then induced MSCs were collected for further experiments.

\section{Choice of differentially expressed miRNAs list using heat map analysis}

We obtained the microarray date from Gene Expression Omnibus (GEO, http://www.ncbi.nlm.nih.gov/geo/), and the GEO accession No. are GSE58027 and GSE89930. The heat map of the miRNAs most obvious differences was created using a method of hierarchical clustering by GeneSpring GX, version 7.3 (Agilent Technologies, Santa Clara, CA, USA)[33].

\section{Data mining in Oncomine database}

The Oncomine database (https ://www.oncom ine.org/resou rce/login.html) is a publicly accessible, online cancer microarray database that helps facilitate research from genome-wide expression analysis[34]. We used the Oncomine database to determine the transcription level of the PIK3R1 gene in sarcoma by retrieving expression levels of PIK3R1 mRNA (log2-transformed) in sarcoma 
vs normal tissues for statistical comparison.

\section{PKH26-labeled exosome and confocal microscopy}

MSC-derived exosomes were labeled by PKH26 and OS cells nuclei were labeled with 4',6-diamidino-2-phenylindole (DAPI) and observed the process of OS cells uptake of exosome under Nikon Eclipse 80i confocal fluorescence microscopy.

\section{Cell proliferation assays}

After reaching $80 \%$ confluence, OS cells were seeded into 96 well plates $(100 \mu \mathrm{L} /$ well $)$ at a density of $6 \times 10^{3}$ cells per well.Cell growth was measured with $10 \mu \mathrm{L}$ cell counting kit $\quad$ (ZP328;

ZOMANBIO, China) according to the manufacturer's instruction. Absorbance was read at $450 \mathrm{~nm}$ using a microplate reader (Tecan Infinite 200 Pro, Shanghai, China). OS cells labeled with CFSE, then OS cells were co-cultured with different culture medium according to the needs of the experiment. OS cells were collected at 24 and $48 \mathrm{hrs}$ and detected by flow cytometry (Becton Dickinson, US) according to the manufacturer's instruction.

\section{wound-healing assay}

OS cells were seeded in a 6-well plate at $1 \times 10^{5}$ cells per well and incubated at $37^{\circ} \mathrm{C}$ in a humidified environment containing $5 \% \mathrm{CO}_{2}$. When cells were grown to $90 \%$ confluence, monolayers were wounded by a sterile $10 \mu \mathrm{L}$ plastic micropipette tip, washed, and added different culture medium according to the needs of the experiment. the width of the scratch gap is viewed under the microscope and photographed.

\section{Transwell invasion assay}

OS cell invasion experiments were conducted using 24-well chambers with 8.0- $\mu \mathrm{m}$ PET membrane pores (Corning Incorporated). The number of cells invading through the membrane was counted in 10 fields/well using an Olympus inverted microscope[35].

\section{Luciferase reporter assays}

OS cells were transfected with different combinations of miR-21-5p mimics/inhibitor or the control sequence, psiCHECK-2-PIK3R1 3'UTR-WT and psiCHECK-2-PIK3R1 3'UTR-Mut for $48 \mathrm{hr}$. The relative luciferase activities were evaluated using the Dual-Luciferase Reporter Assay Kit (Promega Corporation, Madison, WI, USA)[36].

\section{RNA extraction and real-time PCR analysis}

RNA extraction and real-time PCR analysis were performed as described previously[37]. For microRNA quantification, total RNA was reversetranscribed by the TaqMan MicroRNA Reverse Transcription (RT) Kit (Applied Biosystems, Foster City, CA) and then subjected to Taqman miRNA assay (Applied Biosystems) according to the manufacturer's protocol. MiR-21-5p RT: 5'-CTCAACTGGTGTCGTGGAGTCGGCACTGCATACGACTCAACATC-3'. U6 was used as the internal reference for qRT-PCR. The primer sequences are shown in Table 1.

Table 1 The primer sequences for RT-qPCR

\begin{tabular}{|l|l|}
\hline Gene & Sequence \\
\hline miR-21-5p & $\begin{array}{l}\text { Forward primer:5'-ACACTCCAGCTGGGTAGCTTATCAGACTGA-3' } \\
\text { Reverse primer:5'-TGGTGTCGTGGAGTCG-3' }\end{array}$ \\
\hline PIK3R1 & $\begin{array}{l}\text { Forward primer:5'-CGTTTTGGCTGACGCTTTCA-3' } \\
\text { Reverse primer:5'-GTCCCGTCTGCTGTATCTCG-3' }\end{array}$ \\
\hline U6 & $\begin{array}{l}\text { Forward primer:5'-CTCGCTTCGGCAGCACA-3' } \\
\text { Reverse primer:5'-AACGCTTCACGAATTTG CGT-3' }\end{array}$ \\
\hline
\end{tabular}




\section{Western blotting}

Cell lysates were obtained with RIPA lysis buffer (Thermo Fisher Scientific). Protein extraction and western blotting were performed as described previously[38]. The following antibodies were used according to the manufacturer's instructions: Calreticulin (CST, Danvers, MA, USA), CD63 (CST). Specific protein bands were detected using the Chemiluminescent Substrate System (Thermo Fisher Scientific). Proteins were detected using specific antibodies (Abcam, Inc. Shanghai, China). $\beta$-actin (Sigma-Aldrich Co, St Louis, MO, USA) was used as a loading control.

\section{Statistical analyses}

Data were presented as mean \pm standard errors of the means and statistical analyses were performed with IBM SPSS Statistics 21 software and GraphPad Prism 5.0 software. P-values < 0.05 were considered to be statistically significant. All experiments were performed using at least three independent experiments.

\section{Result}

\section{MSCs and MSC-derived exosomes Characterization}

MSCs from bone marrow of patients diagnosed with OS began to adhere to the wall after inoculation 24 hours, and after 2-3 passages MSCs display a fibroblast-like, spindle shaped morphology (Fig.1A), MSCs expressed CD29, CD90, CD44, CD73 and CD105 markers, while they were negative for CD19 and CD133 (Fig.1B). After 1-2 weeks of osteogenic and lipogenic induction, MSCs differentiated into osteocytes and adipocytes which were detected by alizarin red S staining and oil red O staining (Fig.1C). Substantial amounts of MSC- derived exosomes from culture supernatant have been demonstrated by transmission electron microscopy as typical small round nanoparticles with a diameter ranging from 40 to $80 \mathrm{~nm}$ (Fig. 1D). MSC-derived exosomes express primarily surface marker CD63, Calreticulin, an intracellular contaminant, was negatively express (Fig. 1E).

A

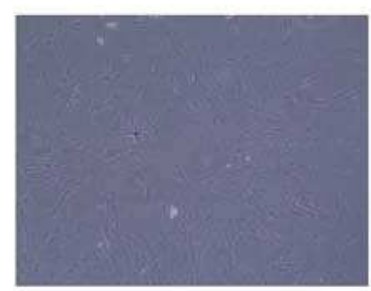

B
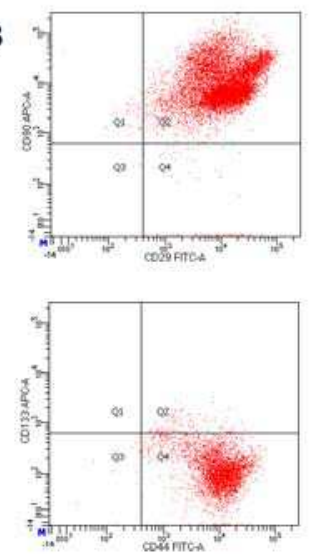
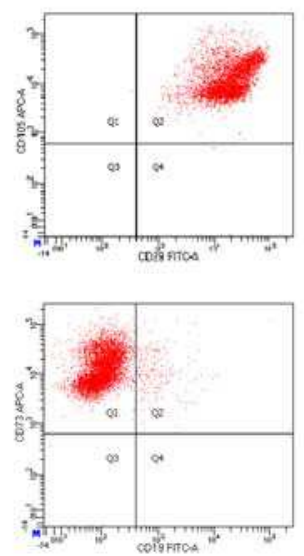

c

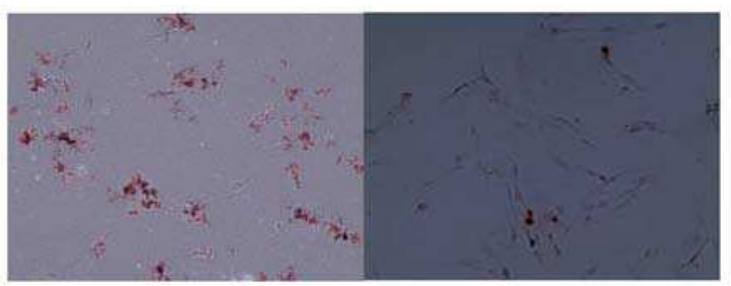

D

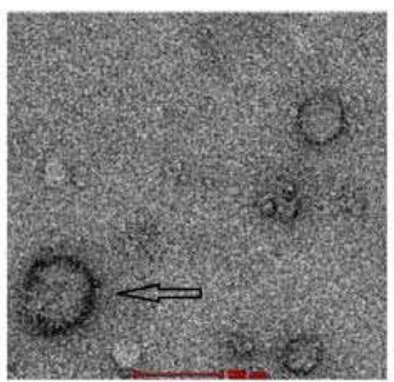

E

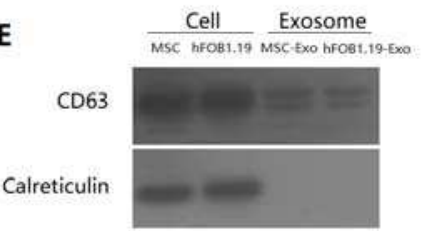


Fig.1 Characterizations of human bone marrow MSCs and MSC-derived exosomes. (A)The cell morphology of human bone marrow MSCs was observed at 3rd passage ; grew as a fibroblast-like, spindle shaped morphology of cells were represented under light microscope. (B) Human bone marrow MSCs phenotypes detected by FACS analysis, showing positive expression of CD29, CD90, CD44, CD73 and CD105 markers, while they were negative for CD19 and CD133. (C) Oil red O staining showed that a small amount of lipid droplets in MSCs display bright red under microscope, and a large number of orange red calcium deposits were observed after alizarin red S staining. (D) Transmission electron microscopy images of exosomes derived from MSCs revealed typical small round nanoparticles with a diameter ranging from 40 to $80 \mathrm{~nm}$. The scale bars indicate $100 \mathrm{~nm}$. (E) Exosomal positive markers CD63 was detected in hBMSC-derived and hFOB1.19 cell-derived exosomes using western blot, whereas negative marker calreticulin was not.

\section{Characterization of exosome internalization by OS cells}

To investigated whether MSC-derived exosomes was internalized by OS cells, we used the fluorescent dye, PKH26, to labeled MSC-derived exosomes, and OS cell nuclei were dyed by DAPI. Under the confocal laser microscope, most of OS cells could see red fluorescence signal, the fluorescent exosomes are mainly located in the cytoplasm of OS cells (U2OS and MG63). For excluding the PKH26 dye contamination in OS cells, exosome-free supernatant was also dyed by PKH26 and no red fluorescence was detected when OS cells (U2OS and MG63) were treated with this control medium(Fig.2).

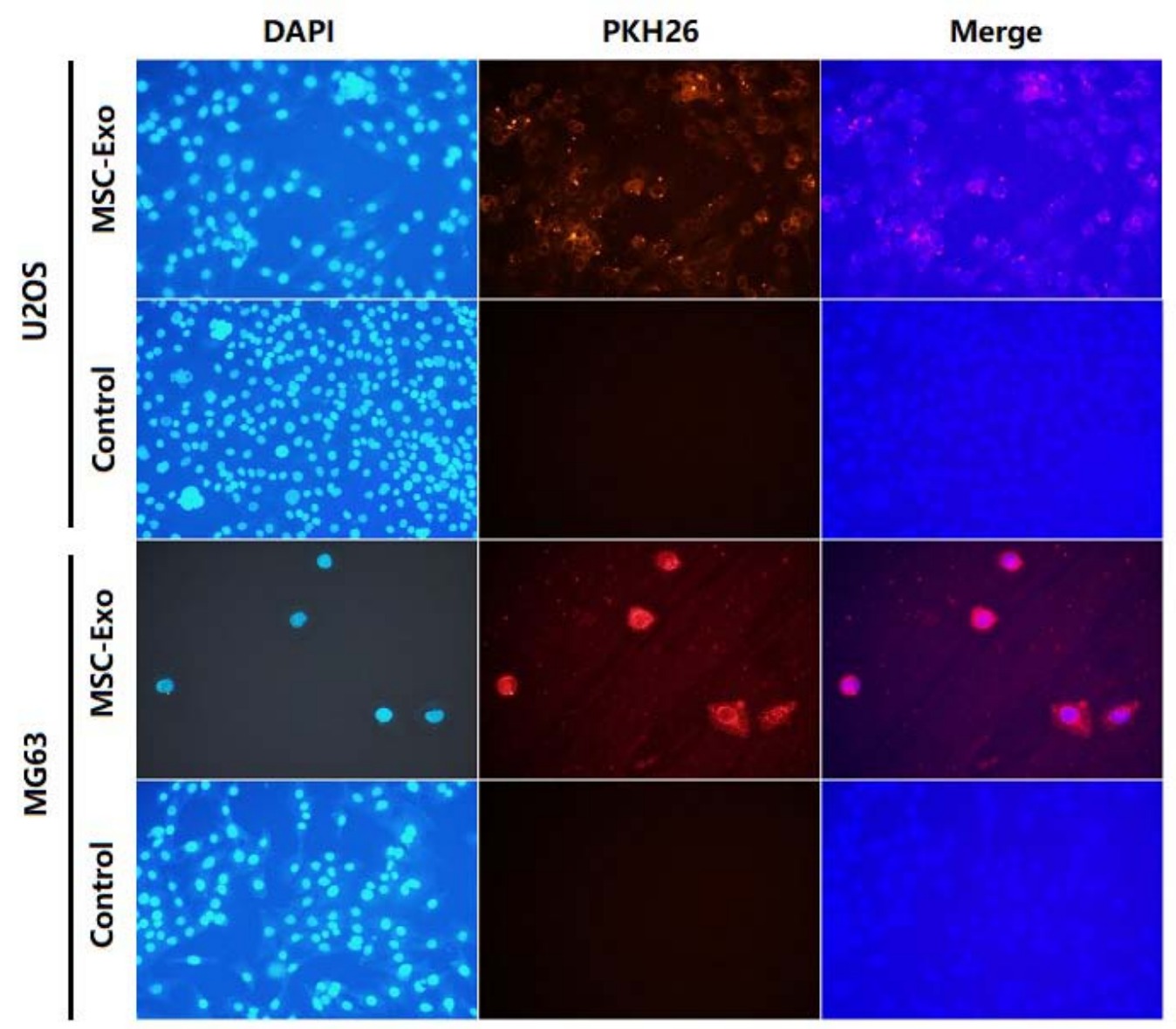

Fig.2 Uptake of hBMSC-exosomes by U2OS and MG63. MSC-derived exosomes were labeled with PKH26 (red) for 4 minutes at room temperature $(\mathrm{B}, \mathrm{H})$, exosome-free supernatant was also dyed by PKH26 as a negative control (E,K). U2OS and MG63 were incubated with absence (control) or presence of labeled 
exosomes for 24 hours, U2OS and MG63 were stained using DAPI (A,D,G,J). PKH26-labelled exosomes have been internalized by U20S and MG63 imaging with confocal fluorescence microscopy(original magnification, $\times 100)(C, I)$.

\section{MSC-derived exosomes promote OS cells proliferation and invasion}

In order to investigate the effect of MSC-derived exosomes on the proliferation of OS cells (U2OS, MG63) in vitro, OS Cells were measured at 24, 48 and 72hr after MSC-derived exosomes and MSC-GW4869 (blockade of exosome generation) treatment. The CCK-8 assay showed that compare with normal group (untreated group), the proliferation rates of both U2OS and MG63 cells were higher after MSC-derived exosomes treatment for $72 \mathrm{hrs}(\mathrm{P}<0.05$, Fig.3A). Therefore, it is suggested that MSC-derived exosomes increased OS cells proliferation. These results were further validated by CFSE fluorescence labeling system. After $72 \mathrm{hrs}$ of co-culture, the proliferation of OS cells (U2OS, MG63) was evaluated by CFSE fluorescence labeling system. The results showed that: the proportions of U2OS and MG63 proliferation in normal group were separately $(65.72 \pm 1.33,62.6 \pm 1.41) \%$, in MSC-Exo group were $(89.64 \pm 2.14,93.40 \pm 1.75) \%$, in MSC-GW4869 group were $(54.95 \pm 4.39,62.8 \pm 4.75) \%$. There were statistical differences between MSC-Exo group and normal group $(\mathrm{P}<0.05)$, while there were no difference between MSC-GW4869 group and normal group ( $(\mathrm{P}>0.05$, Fig.3B). Scratch wound healing assay were conducted to evaluate the effect of MSC-derived exosomes on the invasive abilities of U2OS and MG63. Although no obvious differences were observed between MSC-GW4869 groups and normal groups at 48hours, the rate of wound healing in U2OS and MG63 were significantly accelerated in the MSC-Exo groups at 24, $48 \mathrm{hr}(\mathrm{P}<0.001$,Fig.3 C). These results were further validated by Transwell assays. Compared with MSC-GW4869 groups and normal groups, the number of transmigrated U2OS and MG63 were significantly increased 1.283- and 1.187-fold, respectively, after co-culture with MSC-derived exosomes for 24 hours ( $\mathrm{P}<0.05$, Fig.3 D). To study the protein changes of proliferation and apoptosis of U2OS and MG63, Western blot analysis found compared with MSC-GW4869 and normal group, MSC-derived exosomes can up-regulate the expression of Bcl-2 in U2OS and MG63, while the expression of Bax were down-regulated. These results indicated that MSC-derived exosomes can effectively promote OS cells invasion and proliferation. 
A

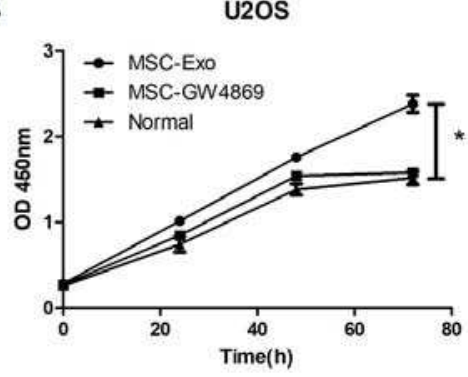

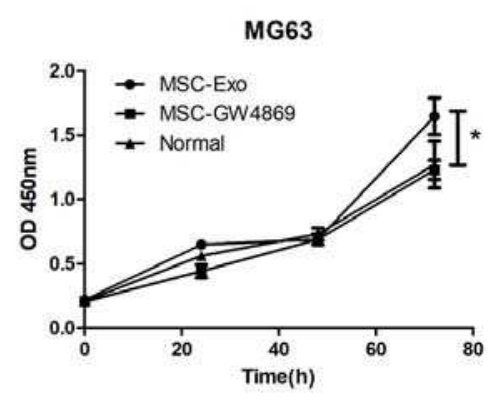

B

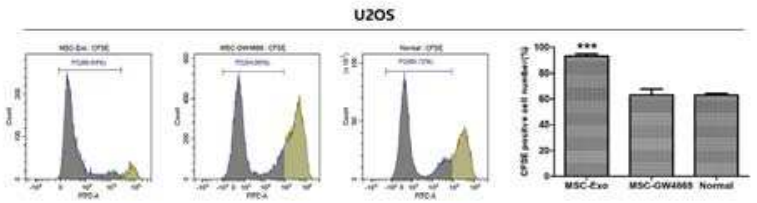
MG63
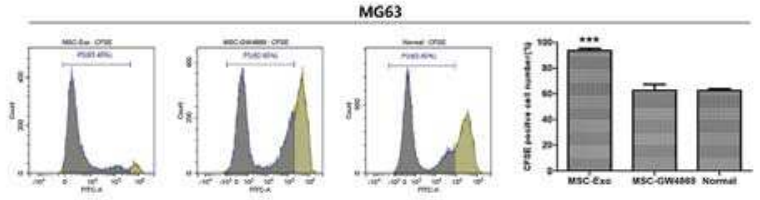

E

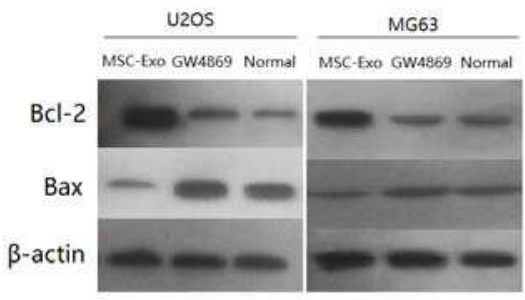

C

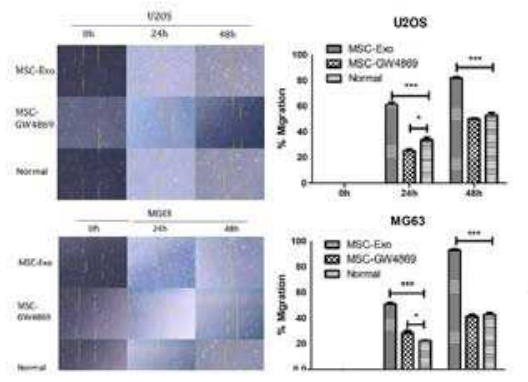

D

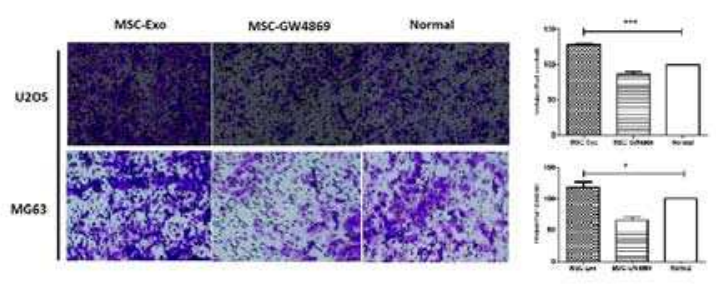

Fig.3 MSC-derived exosomes promote OS cells proliferation and invasion in vitro. (A)U2OS and MG63 were respectively co-cultured with MSC-derived exosomes and MSC- GW4869 for 24, 48 and 72 hours and then subjected to CCK-8 analyses. Compare with normal group (untreated group), the proliferation rates of both U2OS and MG63 cells were higher after MSC-derived exosomes treatment at 72 hours $(\mathrm{P}<0.05)$, whereas there were no significant difference between MSC-GW4869 group and normal group ( $\mathrm{P}>0.05)$. (B)From CFSE fluorescence labeling system, the proportions of U2OS and MG63 proliferation in normal group were separately $(65.72 \pm 1.33$, $62.6 \pm 1.41) \%$, in MSC-Exo group were (89.64 $\pm 2.14,93.40 \pm 1.75) \%$, in MSC-GW4869 group were (54.95 $\pm 4.39,62.8 \pm 4.75) \%$. There were statistical differences between MSC-Exo group and normal group ( $\mathrm{P}<0.001)$, while there were no difference between MSC-GW4869 group and normal group ( $(\mathrm{P}>0.05)$.(C)Scratch wound healing assay test of interfering MSC-derived exosomes group, interfering MSC-GW4869 group and normal group at 24 and 48 hours. The wound healing assay demonstrated a stronger migration ability of U2OS and MG63 in interfering MSC-derived exosomes group. Compared with the normal group and interfering MSC-GW4869 group, there were significant differences in percentage of wound closed at 24 and 48hours $(\mathrm{P}<0.001)$, a weaker migration ability of cells in MSC-GW4869 group and normal group.(D)After 24 hours the number of U2OS and MG63 migrated to the lower chamber of the $8 \mu \mathrm{m}$ pore-sized membrane were analyzed by taking photos and counting the number of cells per visual field. Compared with MSC-GW4869 groups and normal groups, the number of U2OS and MG63 were significantly increased 1.283- and 1.187-fold, respectively. $(\mathrm{P}<0.05)$. (E)The protein levels of Bcl-2 and Bax in U2OS and MG63 cells of various groups which were evaluated by Western blot.Quantitative data from three independent experiments are shown as the mean $\pm \mathrm{SD}$ (error bars). $* \mathrm{P}<0.05$, $* * * \mathrm{P}<0.001$. (Student's 
t-test)

\section{Bioinformatics prediction of exosomal miR-21-5p and targeting gene PIK3R1}

After co-culture of MSC-derived exosomes with OS cell, the proliferation and invasion of OS cells increased. We predict that MSC-derived exosomes, as biological vehicle, play a vital role in interaction between MSCs and OS. To further study the different expression of miRNA (miR) in MSC-derived exosomes, The miRNA profiling by array are available in the Gene Expression Omnibus (GEO) database(login No.GSE58027,GEO, (http://www.ncbi.nlm.nih.gov/geo/) . Compared with adult fibroblasts cells (AFB), miR-21-5p exhibited highly up-regulated expression in MSC-derived exosomes and MSCs (Fig.4A left). From Gene Expression Omnibus (GEO) database (login No. GSE89930), we also found miR-21-5p highly expression between OS cells(Fig.4A right). miR-21-5p plays the role of oncogene in human cancers[19], according to the prediction of miranda, targetscan, encori and pictar, we found there was a miR-21-5p binding site in the 3'noncoding region of PIK3R1 mRNA (Fig.4B). Pan-Cancer analysis from encori database found there was highly negative correlation between miR-21-5p and PIK3R1 in sarcoma (Person $r=-0.443, P=5.31 E-14 ; y=-0.4503 X+10.7515)$ (Fig.4C).

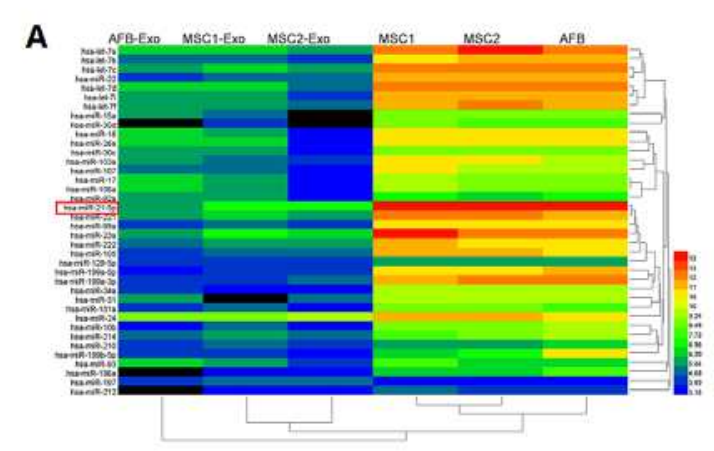

B

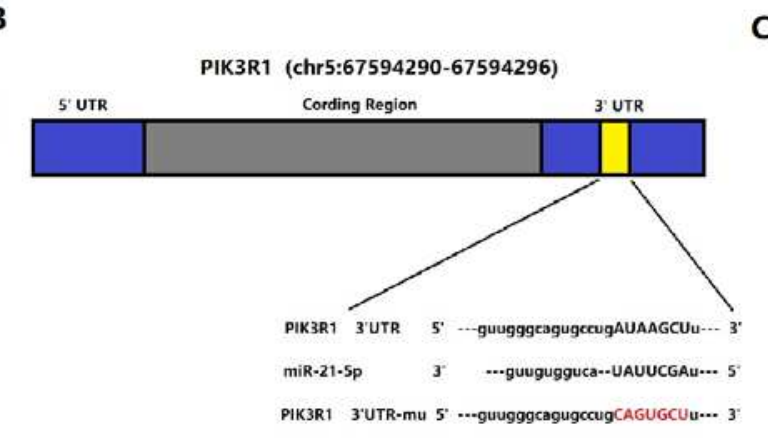

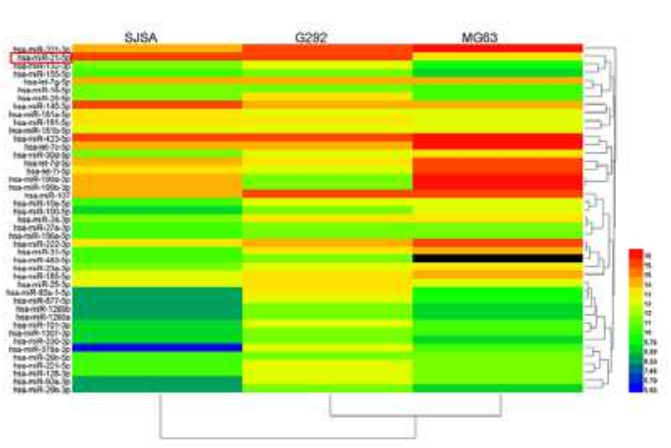

C

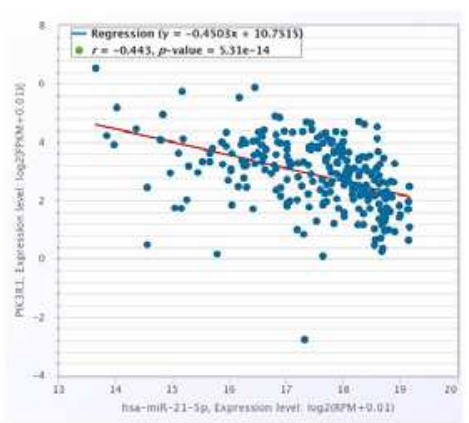

Fig.4 miRNA expression in human MSC and OS cells. (A) Microarray data obtained from the Gene Expression Omnibus database (Accession No. GSE58027, GSE89930). Heat map shows significant upexpression of miR-21-5p in MSCs, MSC-Exo and OS. (blue represents low expression, red represents high expression and black indicates no significant change in gene expression. A color change from blue to red indicates upregulation and a color change from red to blue indicates downregulation). (B) Schematic diagram of the miR-21-5p binding site on PIK3R1, bioinformatics analysis using miranda, targetscan, encori and pictar indicated that the 3'UTR of PIK3R1 mRNA contains a complementary site for the seed region of miR-21-5p. (C) Pan-Cancer analysis found there was highly negative correlation between miR-21-5p and PIK3R1 in sarcoma.

\section{Down-regulation of PIK3R1 mRNA expression in human sarcoma and high-regulated expression of miR-21-5p in MSC-derived exosomes and OS cells}


We analysed the expression of PIK3R1 in human different sarcomas using Oncomine database. cancer vs normal samples in different patient datasets revealed that PIK3R1 expression was significantly lower in pleomorphic myxofibrosarcoma, myxofibrosarcoma, myxoid/round cell liposarcoma, leiomyosarcoma, and malignant fibrous histiocytoma (Fig.5A).

To determine whether MSC-derived exosomes contributed to aberrant expression of miR-21-5p, we perform qRT-PCR to verification. The results indicated that the expression of MSCs and MSC-derived exosomes' miR-21-5p were significantly higher than that of hFOB1.19 cell ( $\mathrm{P}<$ 0.05) (Fig. 5B). On the other hand, we use qRT-PCR to detection of miR-21-5p expression in U2OS and MG-63. After co-culture with MSC-exosomes and MSC-GW8469 for $48 \mathrm{hrs,} \mathrm{the}$ miR-21-5p expression were correspondingly changed in U2OS and MG63.Compared with the normal group (OS cells without treatment), the expression of miR-21-5p in U2OS and MG63 were significantly upregulated after co-culture with MSC-derived exosomes. Moreover, the expression of miR-21-5p in the MSC-GW8469 group have no statistical difference compared with the expression in normal group(Fig.5C). These results indicated that miR-21-5p expression in OS can be effectively modulated through MSC-derived exosomes.

A
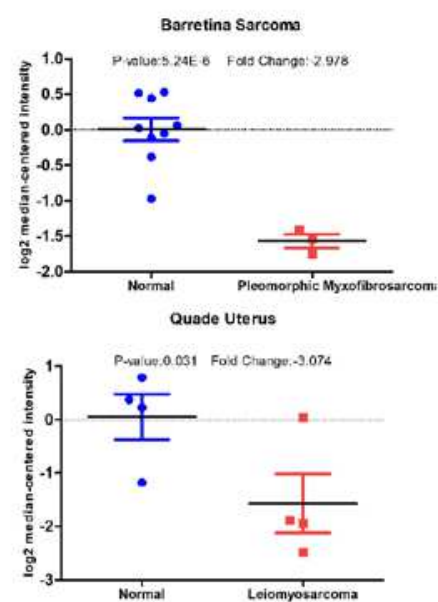

B

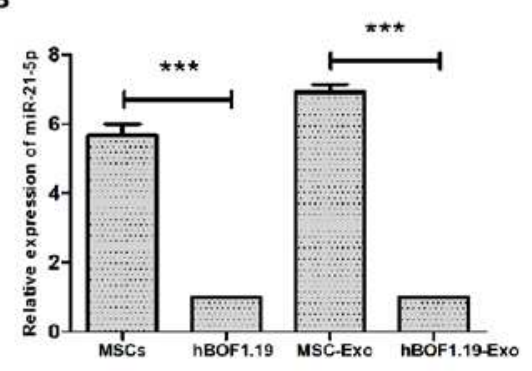

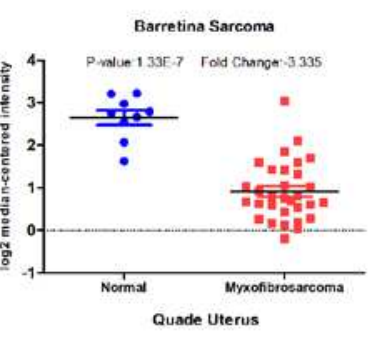
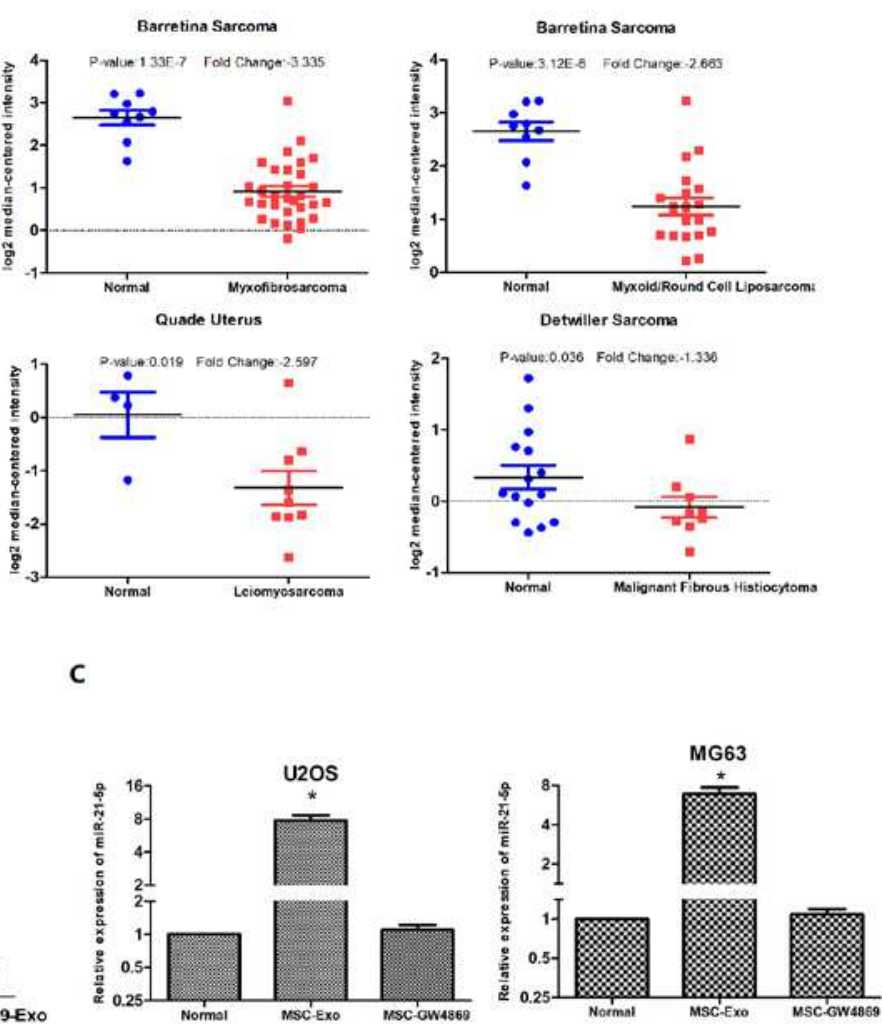

Fig.5 The expression of PIK3R1 are downregulated in human sarcomas and the expression of miR21 $-5 \mathrm{p}$ are upregulated in MSCs, MSC-derived exosomes and OS cells. (A) Analysis of PIK3R1gene expressions in different subtypes of sarcomas using the Oncomine database. Box plot derived from gene expression data in the Oncomine database comparing the expressions of PIK3R1 between normal tissues and cancer tissues in different subtypes of sarcomas, pleomorphic myxofibrosarcoma, myxofibrosarcoma, myxoid/round cell liposarcoma, leiomyosarcoma, and malignant fibrous histiocytoma.(B) The expression of mił21 -5p in MSCs, hFOB1.19 and their purified exosomes examined by RT-qPCR. (C) The expression of miR-21-5p in U2OS and MG63 transfected with MSC-derived exosomes and MGC-GW8469 for 48 hours examined by RT-qPCR. Quantitative data from three 
independent experiments are shown as the mean $\pm \mathrm{SD}$ (error bars). $* \mathrm{P}<0.05$, ***P $<0.001$ (Student's t-test).

Exosomal miR-21-5p derived from MSCs can accelerate OS cell proliferation and invasion To verify the prediction of bioinformatics analysis that MSCs might enhance the proliferation and invasion of OS cells by transferring exosomal miR-21-5p into OS cells, MSCs were treated with miR-21-5p inhibitor and miR-21-5p mimics for $24 \mathrm{hrs}$, then purified the MSC-derived exosomes, which were treated with U2OS and MG63 for 24hrs. The levels of miR-21-5p were quantified using qRT-PCR. The results showed that the level of miR-21-5p in U2OS and MG63 cultured with MSC-derived exosomes (MSC treated with miR-21-5p inhibitor) were much lower compared to that in normal group ( $\mathrm{p}<0.05$ ), and the level of miR-21-5p in U2OS and MG63 internalized with MSC-derived exosomes (MSCs treated with miR-21-5p mimic) was much higher compared to that in normal group ( $\mathrm{p}<0.001)$. Additionally, we failed to find significantly altered miR-21-5p in U2OS and MG-63 cells between the negative groups and the normal groups ( $>0.05$; Fig.6A). In the following experiments, we explored whether MSCs enhance the proliferation and invasion of OS cells by transferring exosomal miR-21-5p. The proliferative abilities of OS cells (U2OS and MG63) were evaluated by CCK 8 assays. Compared with the normal groups, OS cells internalized with MSC-derived exosomes (MSCs treated with miR-21-5p mimic) remarkably increased $(\mathrm{P}<0.05)$. However, Compared with the normal groups, OS cells internalized with MSC-derived exosomes (MSCs treated with miR-21-5p inhibitor) have no significantly increase $(\mathrm{P} \geq 0.05$, Fig. 6B). The results were further validated by CFSE fluorescence labeling system, the results revealing that OS cells internalized with MSC-derived exosomes (MSCs treated with miR-21-5p mimic) proliferative abilities remarkably increased, compared with the normal groups $(\mathrm{p}<0.05)$, while U2OS and MG63 cells proliferative abilities remarkably decreased when it treated with MSC-derived exosomes (MSCs with miR-21-5p inhibitor) (Fig.6C). Scratch wound healing assay were conducted to evaluate the effect of exosomal miR-21-5p on the invasive ability of OS cells. Compared with that of the normal groups, the rates of wound healing in U2OS and MG63 cells were statistically accelerated in miR-21-5p mimic group ( $p<0.05$ ). Compared with that of the normal groups, the rates of wound healing was significantly attenuate in the miR-21-5p inhibitor group( $p<0.05$, fig. $6 \mathrm{D})$. The above findings suggested that up-regulation of exosomal miR-21-5p expression in MSCs could significantly enhance the proliferation and invasion abilities of OS cells internalizing MSC-derived exosomes, while down-regulation of exosomal miR-21-5p in MSCs could attenuate these abilities of OS. 
A

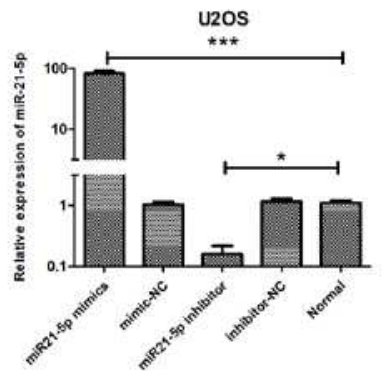

B

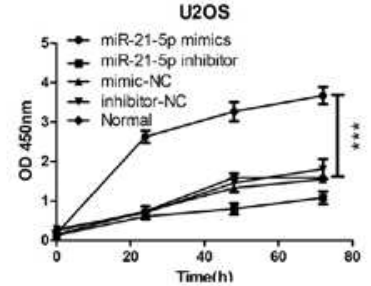

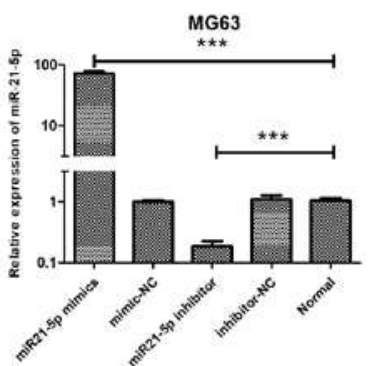

MG63

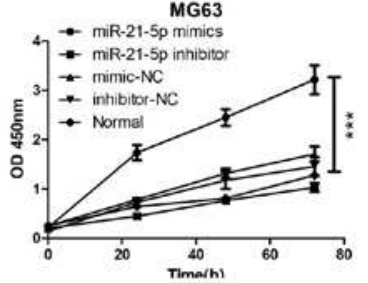

C
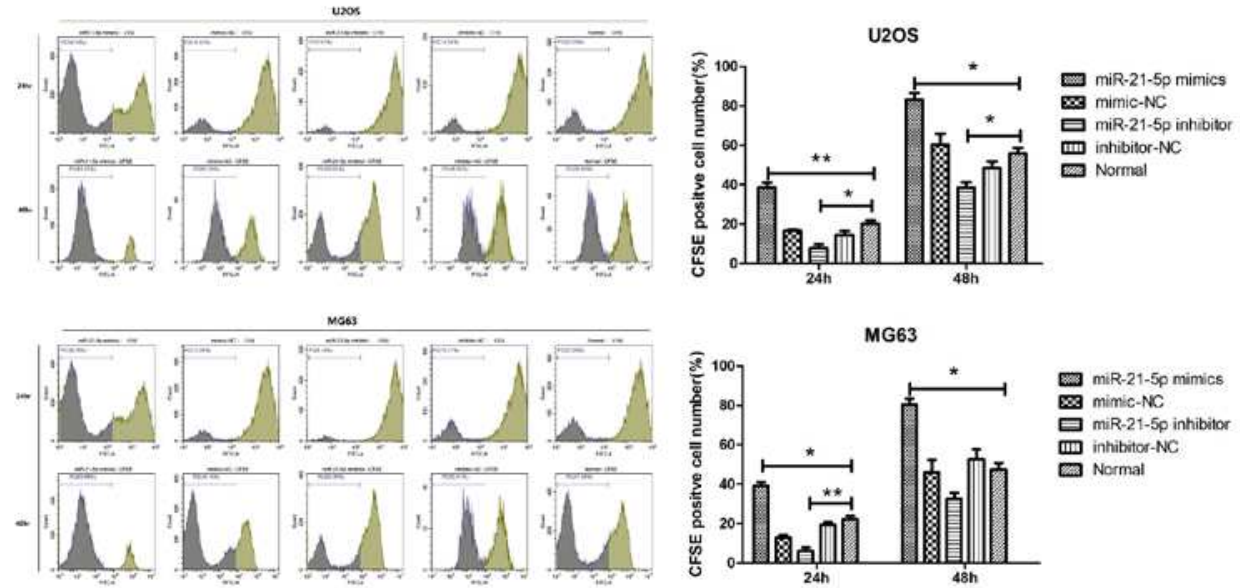

miR-21-5p mimics mimic-NC miR-21-5p inhibitor 西 inhibitor-N

D
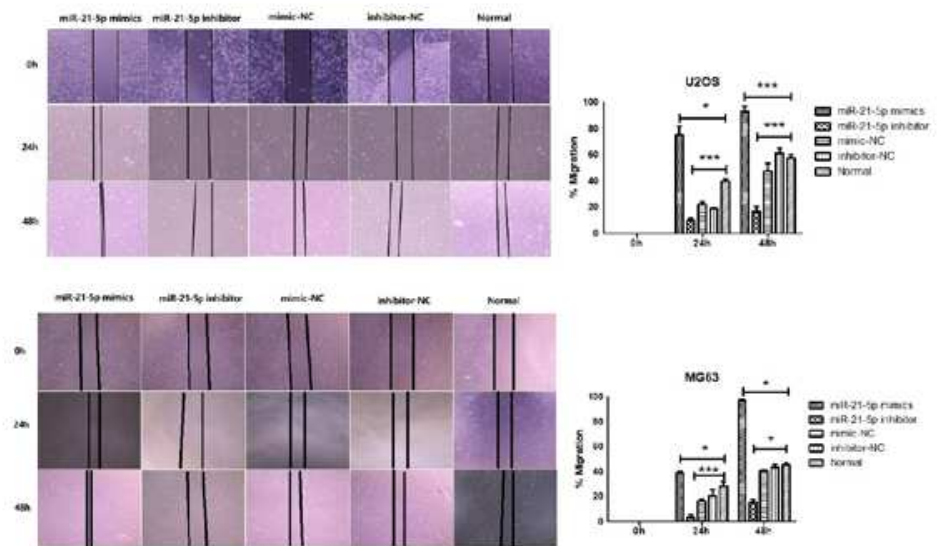

Fig 6. Exosomal miR-21-5p derived from MSCs promotes OS cells proliferation and invasion.(A) Expression of miR-21-5p in cultured U2OS and MG63 cells treated with different exosomes derived from different kinds of MSCs (MSCs treated with synthesized miR-21-5p mimic, miR-21-5p inhibitor, mimic-NC, inhibitor-NC, and without any treatment). (B) U2OS and MG63 cells proliferation following miR-21-5p mimic treatment and miR-21-5p inhibitor examined by CCK-8 assay. (C) CFSE fluorescence labeling system further evaluate U2OS and MG63 cells proliferation in different group. (D) Scratch wound healing assay were further conducted to evaluate the effect of exosomal miR-21-5p on the invasive ability of U2OS and MG63.Quantitative data from 
three independent experiments are shown as the mean $\pm \mathrm{SD}$ (error bars). $* \mathrm{P}<0.05, * * * \mathrm{P}<0.001$. (Student's t-test), miR,microRNA; NC, negative control.

\section{Exosomal miR-21-5p directly targets PIK3R1 to activate PI3K/Akt/mTOR signaling pathway}

To identify the targeting relationship between miR-21-5p and PIK3R1 3'UTR, we perform dual-luciferase reporter assay system and found PIK3R1 was a direct target gene of miR-21-5p (Fig.7A). Moreover, western blot analysis found that miR-21-5p inhibition of MSC-derived exosomes enhanced PIK3R1 protein levels, and miR-21-5p overexpression of MSC-derived exosomes induced the opposite effects in U2OS and MG63 cells. However, there were no significant differences in PIK3R2 expression between groups (Fig.7B). The results of RT-PCR showed the expression level of PIK3R1 have no statistical difference between miR-21-5p inhibitor group and miR-21-5p mimics group (Fig.7C, P>0.05). This suggests that miR-21-5p inhibits the expression of PIK3R1 at post-transcriptional level.

To gain further mechanistic insights into the roles of miR-21-5p/PIK3R1 axis, we hypothesized that exosomal miR-21-5p derived from MSCs activated PI3K/Akt/mTOR signal pathway in OS leading to OS proliferation and invasion, because the PI3K regulatory subunit p85 $\alpha / \mathrm{PIK} 3 \mathrm{R} 1$ can exert tumor suppressor properties through negative regulation of PI3K-Akt signaling pathway[22]. In western blot analysis, the expression levels of p-PI3K, p-Akt and p-mTOR in MSC-derived exosomes -treated OS cells (MSCs treated with miR-21-5p inhibitor ) were prominently depressed compared with controls, while the expression of PIK3R1were significantly increased. After treatment with the MSC-derived exosomes (MSCs treated with miR-21-5p mimics), the expression levels of p-PI3K, p-Akt and p-mTOR in OS cells were all showed a increasing trend, whereas the expression of PIK3R1were sharply decreased $(\mathrm{P}<0.05)$. However the expressions of PI3K, Akt and mTOR were relatively stable in OS cells (Fig.7D).

In summary, The results indicated that bone marrow MSCs could produce amounts of exosomes, which may deliver miR-21-5p into OS cells and thus promote OS proliferation and invasion. The increased miR-21-5p in OS cells specifically targeted the 3UTR region of PIK3R1 mRNA, leading to the decrease of $\mathrm{p} 85 \alpha$ protein level and the activation of corresponding PI3K/Akt/mTOR signaling pathway (Fig. 7E). 
A

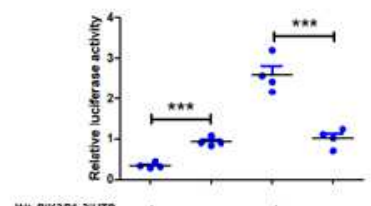

Mt-PIK3R1 SUTR

mil-21-5p irhibititor

B
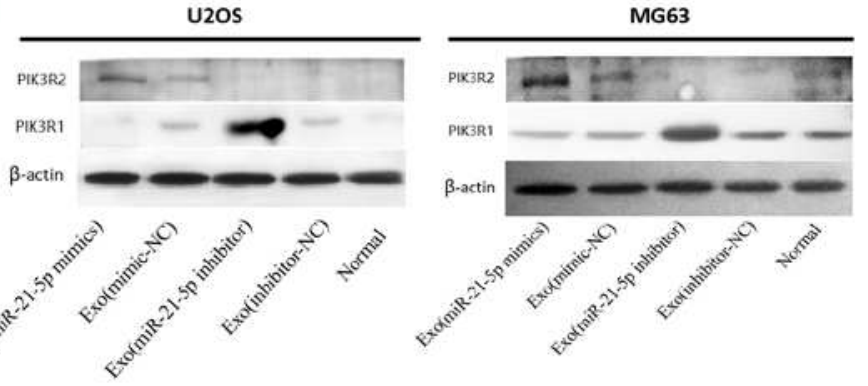

C
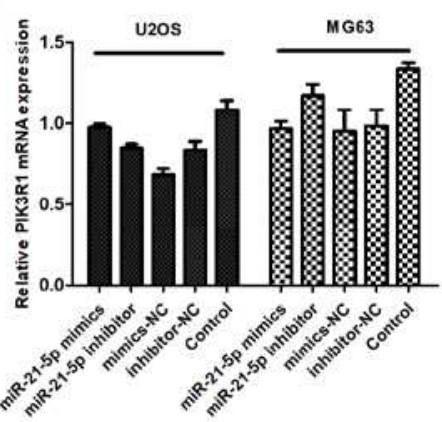

D

U2OS

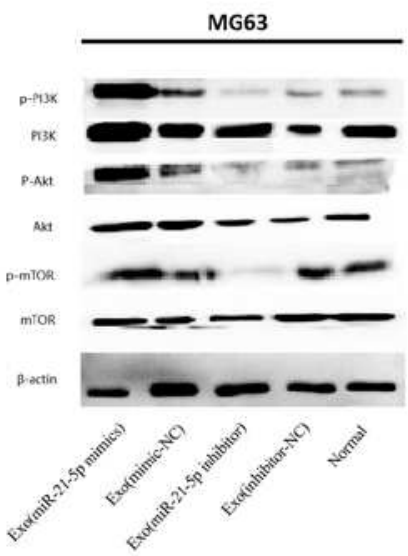

E

MSC (Donor cell) OS cell (Recipient cell)

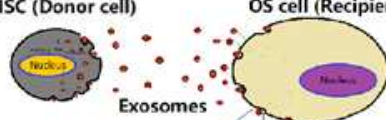

Exosomes

Exosome Growth factor receptor

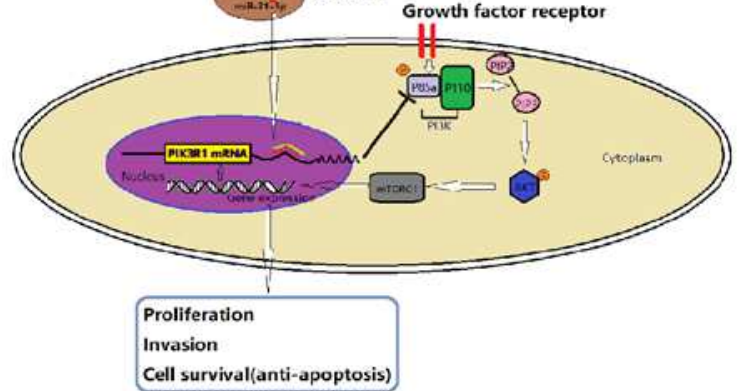


Fig.7 Exosomal miR-21-5p negatively regulated PIK3R1 and then activated the PI3K/AKT/mTOR signal pathway.(A)Luciferase reporter assays were conducted to validate the interaction between miR-21-5p and the 3'UTR of PIK3R1 ( $\mathrm{n}=4$ independent experiments, one-way ANOVA).(B)The western blot assay demonstrated that the protein expression level of PIK3R1 was reduced by miR-21-5p overexpression of MSC-derived exosomes inU2OS and MG63 cells. $\beta$-actin was used as an internal control.(C)T RT-PCR showed the expression level of PIK3R1 have no statistical difference between miR-21-5p inhibitor groups and miR-21-5p mimics groups. (D) Western blot assay was implemented to measure the expression levels of p- or not PI3K, Akt and mTOR in U2OS and MG-63 after treatment vs normal group. All experiments were implemented in triplicate, $* \mathrm{P}<0.05$, ***P<0.001. p-, phosphorylated; wt, wild-type; mt, mutant; NC, negative control.(E) Schematic of the action of exosomal miR-21-5p derived from human bone marrow MSCs regulating OS cells progression. Human bone marrow MSCs transmit miR-21-5p to OS cells through exosomes, exosomal miR-21-5p target PIK3R1 gene of OS cells, then activating PI3K/Akt/mTOR signal pathway and leading to OS proliferation, invasion and anti-apoptosis.

\section{Discussion}

In recent years, accumulating evidence indicates that exosome play an important role in information transfer between cells[15, 39], and released by many cell types when multivesicular endosomes fuse with the plasma membrane[40]. Exosomes are small, lipid bilayer membrane vesicles of 30-100nm diameter and contain a lot of specific biologically active molecules such as lipids, mRNA, regulatory microRNAs, as well as proteins[41]. Studies have shown exosomes from different cell types involved in cancer cells proliferation, angiogenesis, differentiation, metastasis[42-45].

In our previous studies, we found exosomes derived from BMSCs can promote the proliferation and invasion of OS cells. MSCs were used to study the relationship with cancer because MSCs exist in the tumor associated microenvirionment and have a homing ability that allows them to migrate to sites of cancer[46].MSCs are a class of pluripotent cells that can secrete a large number of exosomes which act as paracrine mediators in tumor associated microenvironment. However, the role of MSC-derived exosomes in pathogenesis and progression of cancer cells especially OS has not yet been thoroughly clarified until now. Interestingly, there are contradictory reports that MSCs promote cancer growth. MSC-derived exosomes contain tumor supportive miRNAs, tumor supportive factors, bioactive lipids, lactic acid and glutamic acid, which support breast cancer cell proliferation and metastasis[47]. Whereas MSC-derived exosomes induce tumor associated microenvironment and confer stemness in gastric cancer cells by activating the Akt signaling pathway and serve as a novel mediators of the promoting role of MSCs in gastric[48]. Moreover, study has found that Bone marrow MSCs and Multiple myeloma cells mutually communicate through exosomes, and MSC-derived exosomes enhance Multiple myeloma cell proliferation, migration, and survival and induce drug resistance to bortezomib[49].These authors have demonstrated that MSC-derived exosomes have a role in promoting tumor growth and providing new insight into the interaction of MSCs in tumor development and progression. However, the effects of MSC-derived exosomes on different types of tumor cells proliferation may be completely different. Some reports studied that MSC-derived exosomes inhibit tumor initiation and progression[37, 50,51]. Therefore, it is very important to study the exact mechanism of MSC-derived exosomes on OS proliferation and migration. Our study demonstrated the proliferation and migration effects of MSC-derived exosomes in OS cells and examined which signaling pathway is influenced by MSC-derived exosomes so as to understand the potential 
molecular mechanism on OS.

We first successfully isolated MSCs from bone marrow by adhere-wall culture and bone marrow comes from petients who have been diagnosed as OS. We found that isolated MSCs have the potential of adipogenic and osteogenic differentiation. For further identified we found isolated MSCs expressed CD29, CD90, CD44, CD73 and CD105 markers, while they were negative for CD19 and CD133, this is consistent with the results reported in previous study [38].

The main methods of exosome separation and extraction are gradient ultracentrifugation, kit extraction, ultrafiltration, density gradient centrifugation, polymeric precipitation, immunomagnetic beads. In this experiment, we purified MSC-derived exosomes by gradient ultracentrifugation which has the advantage of high purity. However, this method is complicated, costly and time-consuming and It requires the laboratory to have an ultra-speed centrifuge and its corresponding centrifugal rotor. Following co-culture of OS cells with MSCs, we found that MSCs induced proliferation and invasion of OS cells via delivery of exosomes.

To further examine the mechanism responsible for the effects of MSC-derived exosomes on OS cell proliferation and invasion, we found that in OS associated microenvironment, MSC-exosomes express high level of miR-21-5p, which be regarded as oncogene in cancer[19, 52]. miR-21-5p is frequently overexpressed in various human tumors and in cancer cell lines, and plays an important role in tumorigenesis, high miR-21-5p expression was associated with poor patient performance and shorter survival[19].

Does MSCs in OS associated microenvironment involved in OS proliferation and invasion by mediating exosomal miR-21-5p? At present, it has not been studied. We found transfection of miR-21-5p mimics or miR-21-5p inhibitor to MSCs can significantly up-regulate or down-regulate the expression of miR-21-5p in OS cells and can significantly promote or attenuate the proliferation and invasion of OS. High expression of miR-21-5p in primary OS can be used as an indicator of diagnosis and prognosis of OS[53]. Importantly, we firstly found MSC-derived exosomal miR-21-5p promotes OS proliferation and invasion by targeting PIK3R1 and activity of cancer-related PI3K/Akt/mTOR signaling pathway. PIK3R1 was down-regulated and the $\mathrm{PI} 3 \mathrm{~K} / \mathrm{Akt} / \mathrm{mTOR}$ signaling pathway was promoted in OS cells following treatment of MSC-derived exosomes containing miR-21-5p mimics, and PIK3R1 was up-regulated and $\mathrm{PI} 3 \mathrm{~K} / \mathrm{Akt} / \mathrm{mTOR}$ signaling pathway was inhibited when OS cells treated with MSC-derived exosomes containing miR-21-5p inhibitor.

This study was the first to investigate the relationship between MSC-exosomal miR-21-5p and targeting gene PIK3R1 in OS. Our study has revealed that PIK3R1 is the only down-expressed member of PI3K in OS. PI3K is the key molecule of PI3K/Akt/mTOR signaling pathway which was involved in the regulation of multiple transcription factors in cancer[54]. PI3K are heterodimers composing a regulatory (p85) and catalytic (p110) subunit and exist in four isoforms ( $a, b, c$ and d) with differential tissue expression, according to the different regulatory subunits, class I PI3Ks are divided into three categories, with different structures and functions [55].

PIK3R1 gene encode one of p85-type subunits: p85a[24]. PIK3R1/p85a is the most abundant isoform in normal tissues, but its expression is reduced in cancer[25, 56]. Therefore, PIK3R1/p85a acts as a tumor suppressor because it is capable of maintaining p110a subunit of PI3K in an inactive conformation. Loss of the $\mathrm{p} 85 \alpha$ protein leads to downstream PI3K pathway activation[57-60]. Therefore, the impact of $\mathrm{p} 85 \alpha$ down-regulation on pathway signaling could be caused by the loss of the inhibitory effect of p85 $\alpha$ on p110 and PI3K pathway activity. In spite of 
the extensive knowledge on class I PI3K, until recently, the function and mechanism of PIK3R1 in the proliferation and invasion of malignant tumors are not clear.

In conclusion, this report offers a comprehensive analysis of exosome that sheds light on the cell communication in OS associated microenvironment. The results of our study demonstrate that there were specific conserved sites between miR-21-5p and 3'-UTR of PIK3R1, and PIK3R1 down-regulation by MSC-derived exosomes containing abundant miR-21-5p exerts tumor promoter properties in OS. All these results support the notion that exosomal miR-21-5p derived from MSCs exerts tumor promoter properties in OS by down-regulating PIK3R1 so as to activate of the PI3K/Akt/mTOR signaling pathway.

\section{Acknowledgements}

The authors would like to thank Yali Zhou and Yali Liu for flow cytometry. Flow cytometry assays were performed on a device of Beckman coulter.

\section{Author's contributions}

QJ conceived and designed the experiments. QJ, ZRH performed the experiments. WZL performed bioinformatic analysis. XYY revised the paper. All authors read and approved the final manuscript.

\section{Funding}

This work were supported by Youth Program of National Natural Science Foundation of China (No.31801194), Youth Program of Science and Technology Fund of Gansu (No.18JR3RA325), "Cuiying Science and Technology Innovation" program of Lanzhou University Second Hospital (No.CY2018-QN02) and Innovation Ability Improvement Project of Colleges and Universities in Gansu Province (No.2019B-014).

\section{Availability of data and materials}

All data generated or analyzed during the study are included in this published article. GEO data was downloaded from the GEO datasets.(https://www.ncbi.nlm.nih.gov/gds/).

\section{Ethics approval and consent to participate}

The research approved by the Ethics Committee of Lanzhou university second hospital and informed consent was obtained from all participants included in the study, in agreement with institutional guidelines.

\section{Consent for publication}

Not applicable.

\section{Competing interests}

The authors declare that they have no competing interests.

\section{Author details}

1 Lanzhou University Second hospital, Lanzhou, People's Republic of China, 2 Orthopaedics Key Laboratory of Gansu Province, Lanzhou, Gansu, 730031, People's Republic of China

\section{Reference}


Brown HK, Tellez-Gabriel M, Heymann D: Cancer stem cells in osteosarcoma. Cancer Lett 2017;386:189-195.

2 Le Nail LR, Brennan M, Rosset P, Deschaseaux F, Piloquet P, Pichon O, Le Caignec C, Crenn V, Layrolle P, Herault O, De Pinieux G, Trichet V: Comparison of Tumor- and Bone Marrow-Derived Mesenchymal Stromal/Stem Cells from Patients with High-Grade Osteosarcoma. Int J Mol Sci 2018;19:

3 Pittenger MF, Mackay AM, Beck SC, Jaiswal RK, Douglas R, Mosca JD, Moorman MA, Simonetti DW, Craig S, Marshak DR: Multilineage potential of adult human mesenchymal stem cells. Science 1999;284:143-147.

4 Campagnoli C, Roberts IA, Kumar S, Bennett PR, Bellantuono I, Fisk NM: Identification of mesenchymal stem/progenitor cells in human first-trimester fetal blood, liver, and bone marrow. Blood 2001;98:2396-2402.

5 Gazdic M, Volarevic V, Arsenijevic N, Stojkovic M: Mesenchymal stem cells: a friend or foe in immune-mediated diseases. Stem Cell Rev Rep 2015;11:280-287.

6 Marfia G, Navone SE, Di Vito C, Ughi N, Tabano S, Miozzo M, Tremolada C, Bolla G, Crotti C, Ingegnoli F, Rampini P, Riboni L, Gualtierotti R, Campanella R: Mesenchymal stem cells: potential for therapy and treatment of chronic non-healing skin wounds. Organogenesis 2015;11:183-206.

$7 \quad$ Karp JM, Leng Teo GS: Mesenchymal stem cell homing: the devil is in the details. Cell Stem Cell 2009;4:206-216.

8 Pascucci L, Cocce V, Bonomi A, Ami D, Ceccarelli P, Ciusani E, Vigano L, Locatelli A, Sisto F, Doglia SM, Parati E, Bernardo ME, Muraca M, Alessandri G, Bondiolotti G, Pessina A: Paclitaxel is incorporated by mesenchymal stromal cells and released in exosomes that inhibit in vitro tumor growth: a new approach for drug delivery. J Control Release 2014;192:262-270. Karnoub AE, Dash AB, Vo AP, Sullivan A, Brooks MW, Bell GW, Richardson AL, Polyak K, Tubo R, Weinberg RA: Mesenchymal stem cells within tumour stroma promote breast cancer metastasis. Nature 2007;449:557-563.

Mohseny AB, Szuhai K, Romeo S, Buddingh EP, Briaire-de Bruijn I, de Jong D, van Pel M, Cleton-Jansen AM, Hogendoorn PC: Osteosarcoma originates from mesenchymal stem cells in consequence of aneuploidization and genomic loss of Cdkn2. J Pathol 2009;219:294-305. Cipriani P, Ruscitti P, Di Benedetto P, Carubbi F, Liakouli V, Berardicurti O, Ciccia F, Triolo G, Giacomelli R: Mesenchymal stromal cells and rheumatic diseases: new tools from pathogenesis to regenerative therapies. Cytotherapy 2015;17:832-849.

Toh WS, Lai RC, Zhang B, Lim SK: MSC exosome works through a protein-based mechanism of action. Biochem Soc Trans 2018;46:843-853.

Wu P, Zhang B, Shi H, Qian H, Xu W: MSC-exosome: A novel cell-free therapy for cutaneous regeneration. Cytotherapy 2018;20:291-301.

He X, Dong Z, Cao Y, Wang H, Liu S, Liao L, Jin Y, Yuan L, Li B: MSC-Derived Exosome Promotes M2 Polarization and Enhances Cutaneous Wound Healing. Stem Cells Int 2019;2019:7132708.

Milane L, Singh A, Mattheolabakis G, Suresh M, Amiji MM: Exosome mediated communication within the tumor microenvironment. J Control Release 2015;219:278-294. mechanism of cell-to-cell communication. Kidney Int 2010;78:838-848. 
Joyce DP, Kerin MJ, Dwyer RM: Exosome-encapsulated microRNAs as circulating biomarkers for breast cancer. Int J Cancer 2016;139:1443-1448.

Trohatou O, Zagoura D, Bitsika V, Pappa KI, Antsaklis A, Anagnou NP, Roubelakis MG: Sox 2 suppression by miR-21 governs human mesenchymal stem cell properties. Stem Cells Transl Med 2014;3:54-68.

Pfeffer SR, Yang CH, Pfeffer LM: The Role of miR-21 in Cancer. Drug Dev Res 2015;76:270-277.

Rathinaswamy MK, Burke JE: Class I phosphoinositide 3-kinase (PI3K) regulatory subunits and their roles in signaling and disease. Adv Biol Regul 2020;75:100657.

Shekar SC, Wu H, Fu Z, Yip SC, Nagajyothi, Cahill SM, Girvin ME, Backer JM: Mechanism of constitutive phosphoinositide 3-kinase activation by oncogenic mutants of the p85 regulatory subunit. J Biol Chem 2005;280:27850-27855.

Taniguchi CM, Winnay J, Kondo T, Bronson RT, Guimaraes AR, Aleman JO, Luo J, Stephanopoulos G, Weissleder R, Cantley LC, Kahn CR: The phosphoinositide 3-kinase regulatory subunit $\mathrm{p} 85 \mathrm{alpha}$ can exert tumor suppressor properties through negative regulation of growth factor signaling. Cancer Res 2010;70:5305-5315.

Zhao JJ, Liu Z, Wang L, Shin E, Loda MF, Roberts TM: The oncogenic properties of mutant p110alpha and p110beta phosphatidylinositol 3-kinases in human mammary epithelial cells. Proc Natl Acad Sci U S A 2005;102:18443-18448.

Vallejo-Diaz J, Chagoyen M, Olazabal-Moran M, Gonzalez-Garcia A, Carrera AC: The Opposing Roles of PIK3R1/p85alpha and PIK3R2/p85beta in Cancer. Trends Cancer 2019;5:233-244.

Cortes I, Sanchez-Ruiz J, Zuluaga S, Calvanese V, Marques M, Hernandez C, Rivera T, Kremer L, Gonzalez-Garcia A, Carrera AC: p85beta phosphoinositide 3-kinase subunit regulates tumor progression. Proc Natl Acad Sci U S A 2012;109:11318-11323.

Sun M, Hillmann P, Hofmann BT, Hart JR, Vogt PK: Cancer-derived mutations in the regulatory subunit p85alpha of phosphoinositide 3-kinase function through the catalytic subunit p110alpha. Proc Natl Acad Sci U S A 2010;107:15547-15552.

Song L, Xie X, Yu S, Peng F, Peng L: MicroRNA126 inhibits proliferation and metastasis by targeting pik3r2 in prostate cancer. Mol Med Rep 2016;13:1204-1210.

Hirsch E, Ciraolo E, Franco I, Ghigo A, Martini M: PI3K in cancer-stroma interactions: bad in seed and ugly in soil. Oncogene 2014;33:3083-3090.

Yue Z, Guan X, Chao R, Huang C, Li D, Yang P, Liu S, Hasegawa T, Guo J, Li M: Diallyl Disulfide Induces Apoptosis and Autophagy in Human Osteosarcoma MG-63 Cells through the PI3K/Akt/mTOR Pathway. Molecules 2019;24:

Qi J, Zhou Y, Jiao Z, Wang X, Zhao Y, Li Y, Chen H, Yang L, Zhu H, Li Y: Exosomes Derived from Human Bone Marrow Mesenchymal Stem Cells Promote Tumor Growth Through Hedgehog Signaling Pathway. Cell Physiol Biochem 2017;42:2242-2254.

Dudakovic A, Camilleri E, Riester SM, Lewallen EA, Kvasha S, Chen X, Radel DJ, Anderson JM, Nair AA, Evans JM, Krych AJ, Smith J, Deyle DR, Stein JL, Stein GS, Im HJ, Cool SM, Westendorf JJ, Kakar S, Dietz AB, van Wijnen AJ: High-resolution molecular validation of self-renewal and spontaneous differentiation in clinical-grade adipose-tissue derived human mesenchymal stem cells. J Cell Biochem 2014;115:1816-1828. 
from cell culture supernatants and biological fluids. Curr Protoc Cell Biol 2006; Chapter 3:Unit 322.

33 Saeed AI, Sharov V, White J, Li J, Liang W, Bhagabati N, Braisted J, Klapa M, Currier T, Thiagarajan M, Sturn A, Snuffin M, Rezantsev A, Popov D, Ryltsov A, Kostukovich E, Borisovsky I, Liu Z, Vinsavich A, Trush V, Quackenbush J: TM4: a free, open-source system for microarray data management and analysis. Biotechniques 2003;34:374-378.

Liu Y, Qi J, Dou Z, Hu J, Lu L, Dai H, Wang H, Yang W: Systematic expression analysis of WEE family kinases reveals the importance of PKMYT1 in breast carcinogenesis. Cell Prolif 2020;53:e12741.

Qin F, Tang H, Zhang Y, Zhang Z, Huang P, Zhu J: Bone marrow-derived mesenchymal stem cell-derived exosomal microRNA-208a promotes osteosarcoma cell proliferation, migration, and invasion. J Cell Physiol 2020;235:4734-4745.

Liu ZZ, Tian YF, Wu H, Ouyang SY, Kuang WL: LncRNA H19 promotes glioma angiogenesis through miR-138/HIF-1alpha/VEGF axis. Neoplasma 2020;67:111-118.

Lee JK, Park SR, Jung BK, Jeon YK, Lee YS, Kim MK, Kim YG, Jang JY, Kim CW: Exosomes derived from mesenchymal stem cells suppress angiogenesis by down-regulating VEGF expression in breast cancer cells. PLoS One 2013;8:e84256.

Qu M, Cui J, Zhu J, Ma Y, Yuan X, Shi J, Guo D, Li C: Bone marrow-derived mesenchymal stem cells suppress NK cell recruitment and activation in PolyI:C-induced liver injury. Biochem Biophys Res Commun 2015;466:173-179.

He C, Zheng S, Luo Y, Wang B: Exosome Theranostics: Biology and Translational Medicine. Theranostics 2018;8:237-255.

40 Raposo G, Stoorvogel W: Extracellular vesicles: exosomes, microvesicles, and friends. J Cell Biol 2013;200:373-383.

41 Andreu Z, Yanez-Mo M: Tetraspanins in extracellular vesicle formation and function. Front Immunol 2014;5:442.

42 Ansa-Addo EA, Lange S, Stratton D, Antwi-Baffour S, Cestari I, Ramirez MI, McCrossan MV, Inal JM: Human plasma membrane-derived vesicles halt proliferation and induce differentiation of THP-1 acute monocytic leukemia cells. J Immunol 2010;185:5236-5246.

43 Roccaro AM, Sacco A, Maiso P, Azab AK, Tai YT, Reagan M, Azab F, Flores LM, Campigotto F, Weller E, Anderson KC, Scadden DT, Ghobrial IM: BM mesenchymal stromal cell-derived exosomes facilitate multiple myeloma progression. J Clin Invest 2013;123:1542-1555.

Mineo M, Garfield SH, Taverna S, Flugy A, De Leo G, Alessandro R, Kohn EC: Exosomes released by K562 chronic myeloid leukemia cells promote angiogenesis in a Src-dependent fashion. Angiogenesis 2012;15:33-45.

Tadokoro H, Umezu T, Ohyashiki K, Hirano T, Ohyashiki JH: Exosomes derived from hypoxic leukemia cells enhance tube formation in endothelial cells. $\mathrm{J}$ Biol Chem 2013;288:34343-34351.

46 Spaeth E, Klopp A, Dembinski J, Andreeff M, Marini F: Inflammation and tumor microenvironments: defining the migratory itinerary of mesenchymal stem cells. Gene Ther 2008; 15:730-738.

47 Vallabhaneni KC, Penfornis P, Dhule S, Guillonneau F, Adams KV, Mo YY, Xu R, Liu Y, Watabe K, Vemuri MC, Pochampally R: Extracellular vesicles from bone marrow 
mesenchymal stem/stromal cells transport tumor regulatory microRNA, proteins, and metabolites. Oncotarget 2015;6:4953-4967.

48 Gu H, Ji R, Zhang X, Wang M, Zhu W, Qian H, Chen Y, Jiang P, Xu W: Exosomes derived from human mesenchymal stem cells promote gastric cancer cell growth and migration via the activation of the Akt pathway. Mol Med Rep 2016;14:3452-3458.

49 Wang J, Hendrix A, Hernot S, Lemaire M, De Bruyne E, Van Valckenborgh E, Lahoutte T, De Wever O, Vanderkerken K, Menu E: Bone marrow stromal cell-derived exosomes as communicators in drug resistance in multiple myeloma cells. Blood 2014;124:555-566.

Bruno S, Collino F, Deregibus MC, Grange C, Tetta C, Camussi G: Microvesicles derived from human bone marrow mesenchymal stem cells inhibit tumor growth. Stem Cells Dev 2013;22:758-771.

Han I, Yun M, Kim EO, Kim B, Jung MH, Kim SH: Umbilical cord tissue-derived mesenchymal stem cells induce apoptosis in PC-3 prostate cancer cells through activation of JNK and downregulation of PI3K/AKT signaling. Stem Cell Res Ther 2014;5:54.

Cortez MA, Ivan C, Zhou P, Wu X, Ivan M, Calin GA: microRNAs in cancer: from bench to bedside. Adv Cancer Res 2010;108:113-157.

Ziyan W, Shuhua Y, Xiufang W, Xiaoyun L: MicroRNA-21 is involved in osteosarcoma cell invasion and migration. Med Oncol 2011;28:1469-1474.

54 Zhu JF, Huang W, Yi HM, Xiao T, Li JY, Feng J, Yi H, Lu SS, Li XH, Lu RH, He QY, Xiao ZQ: Annexin A1-suppressed autophagy promotes nasopharyngeal carcinoma cell invasion and metastasis by PI3K/AKT signaling activation. Cell Death Dis 2018;9:1154.

Dienstmann R, Rodon J, Serra V, Tabernero J: Picking the point of inhibition: a comparative review of PI3K/AKT/mTOR pathway inhibitors. Mol Cancer Ther 2014;13:1021-1031.

Alcazar I, Cortes I, Zaballos A, Hernandez C, Fruman DA, Barber DF, Carrera AC: p85beta phosphoinositide 3-kinase regulates CD28 coreceptor function. Blood 2009;113:3198-3208.

Cheung LW, Hennessy BT, Li J, Yu S, Myers AP, Djordjevic B, Lu Y, Stemke-Hale K, Dyer MD, Zhang F, Ju Z, Cantley LC, Scherer SE, Liang H, Lu KH, Broaddus RR, Mills GB: High frequency of PIK3R1 and PIK3R2 mutations in endometrial cancer elucidates a novel mechanism for regulation of PTEN protein stability. Cancer Discov 2011;1:170-185.

58 2584672725846727Martincorena I, Campbell PJ: Somatic mutation in cancer and normal cells. Science 2015;349:1483-1489.

59 Toste PA, Li L, Kadera BE, Nguyen AH, Tran LM, Wu N, Madnick DL, Patel SG, Dawson DW, Donahue TR: p85alpha is a microRNA target and affects chemosensitivity in pancreatic cancer. J Surg Res 2015;196:285-293.

60 Jaiswal BS, Janakiraman V, Kljavin NM, Chaudhuri S, Stern HM, Wang W, Kan Z, Dbouk HA, Peters BA, Waring P, Dela Vega T, Kenski DM, Bowman KK, Lorenzo M, Li H, Wu J, Modrusan Z, Stinson J, Eby M, Yue P, Kaminker JS, de Sauvage FJ, Backer JM, Seshagiri S: Somatic mutations in p85alpha promote tumorigenesis through class IA PI3K activation. Cancer Cell 2009;16:463-474. 


\section{Figures}

A

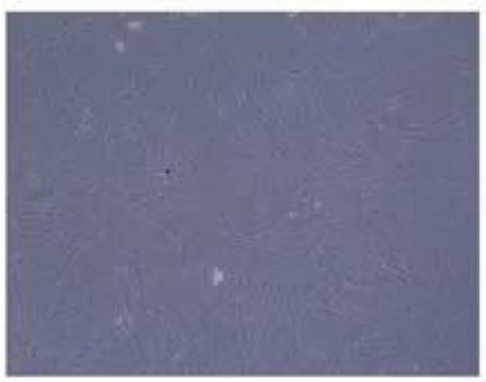

c

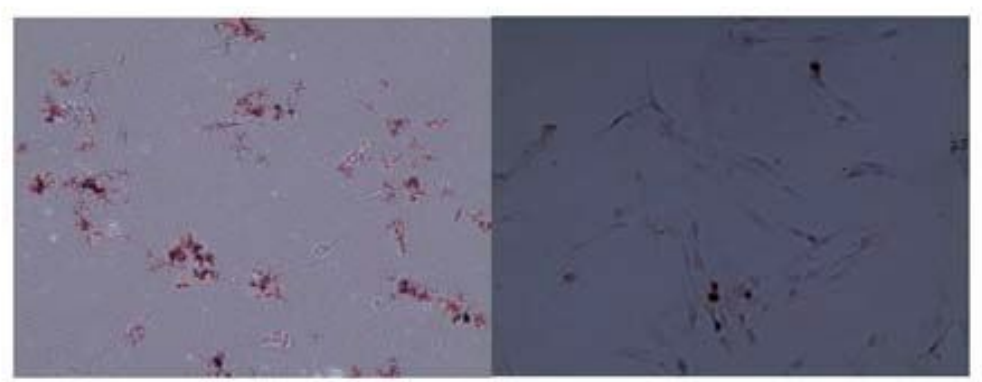

D

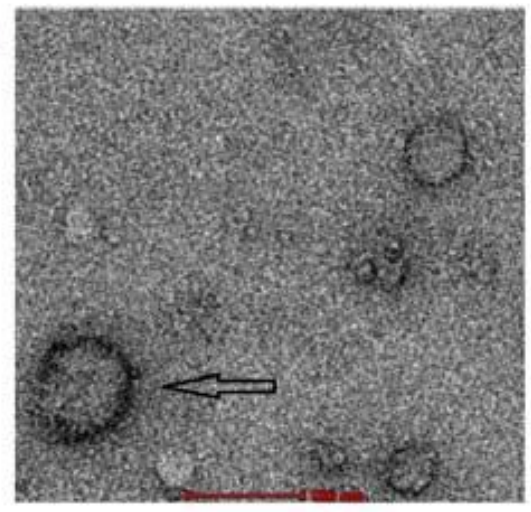

E

Cell Exosome

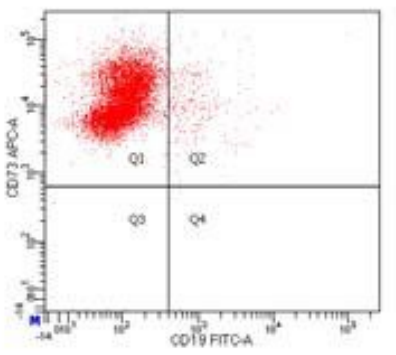
MSC hFOB1.19 MSC-Exo hFOB1.19-Exo

CD63 MSC hFoB1.19 MSC-Exo hFoBt.19-50.
Calreticulin

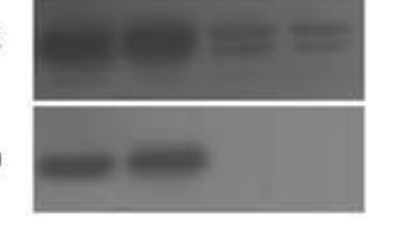

\section{Figure 1}

Characterizations of human bone marrow MSCs and MSC-derived exosomes. (A)The cell morphology of human bone marrow MSCs was observed at 3rd passage ; grew as a fibroblast-like, spindle shaped morphology of cells were represented under light microscope. (B) Human bone marrow MSCs phenotypes detected by FACS analysis, showing positive expression of CD29, CD90, CD44, CD73 and CD105 markers, while they were negative for CD19 and CD133. (C) Oil red O staining showed that a small amount of lipid droplets in MSCs display bright red under microscope, and a large number of orange red calcium deposits were observed after alizarin red S staining. (D) Transmission electron microscopy images of exosomes derived from MSCs revealed typical small round nanoparticles with a diameter ranging from 40 to $80 \mathrm{~nm}$. The scale bars indicate $100 \mathrm{~nm}$. (E) Exosomal positive markers CD63 was detected in hBMSC-derived and hFOB1.19 cell-derived exosomes using western blot, whereas negative marker calreticulin was not. 


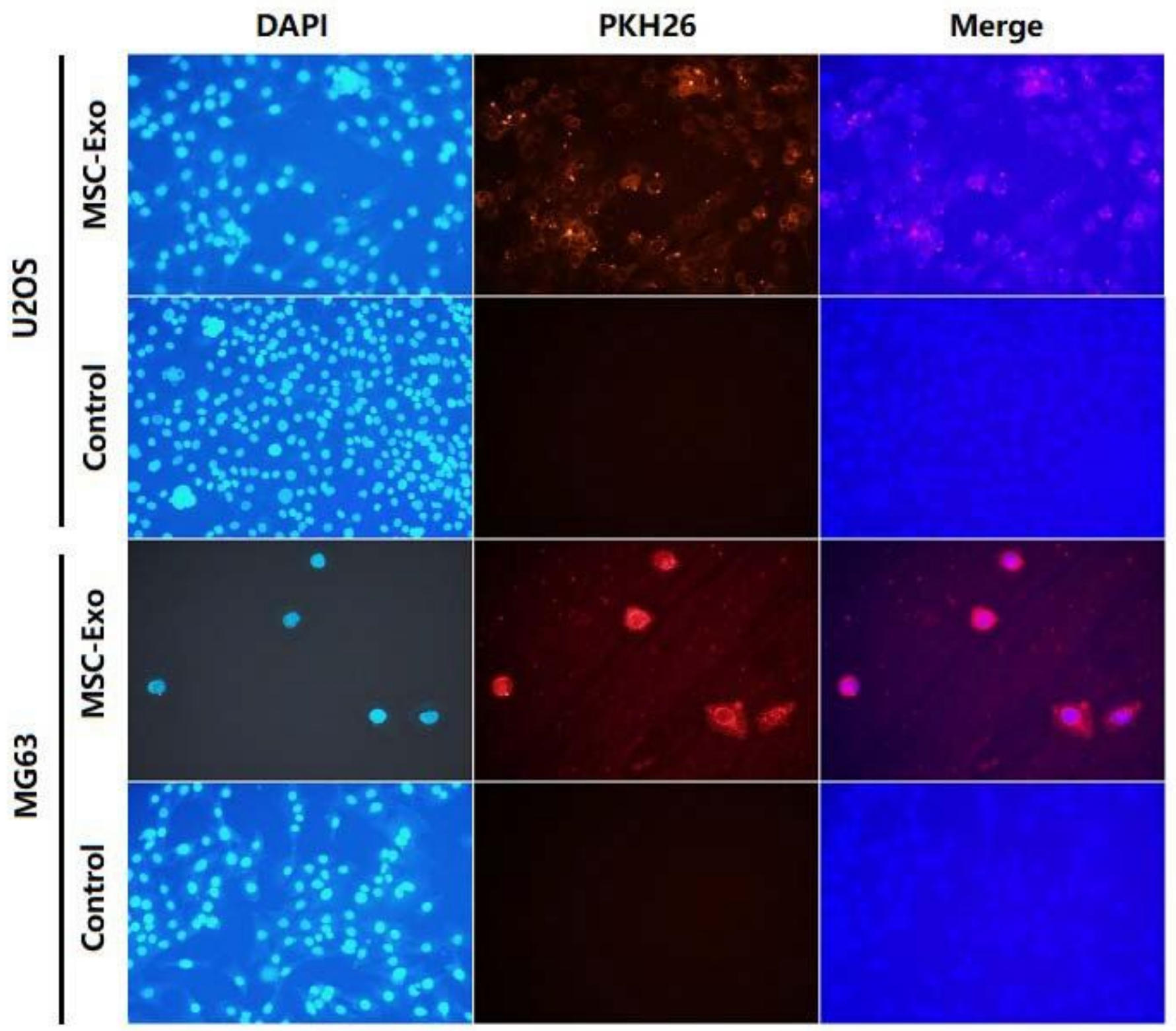

Figure 2

Uptake of hBMSC-exosomes by U2OS and MG63. MSC-derived exosomes were labeled with PKH26 (red) for 4 minutes at room temperature(B,H), exosome-free supernatant was also dyed by $\mathrm{PKH} 26$ as a negative control (E,K). U2OS and MG63 were incubated with absence (control) or presence of labeled exosomes for 24 hours, U2OS and MG63 were stained using DAPI (A,D,G,J). PKH26-labelled exosomes have been internalized by U2OS and MG63 imaging with confocal fluorescence microscopy(original magnification, $\times 100)(C, I)$. 
A

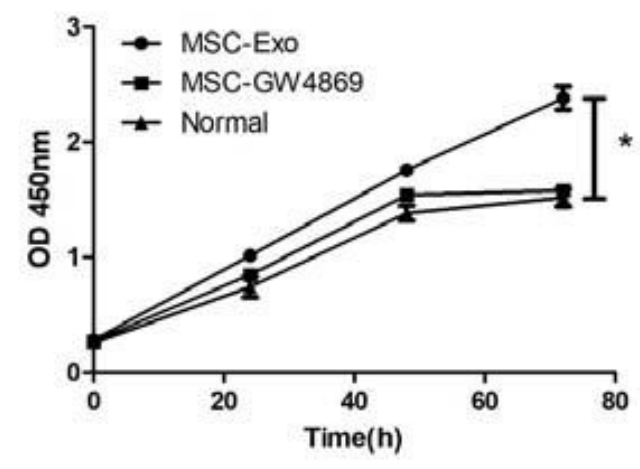

MG63

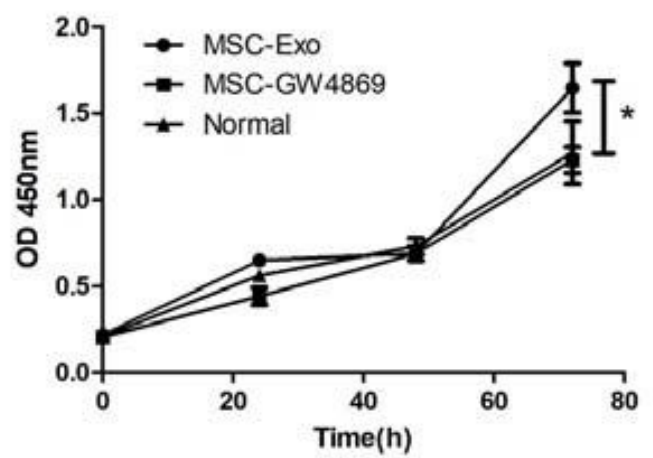

B

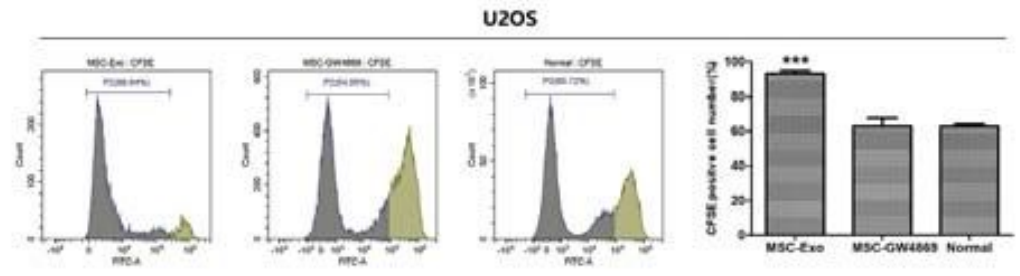

MG63
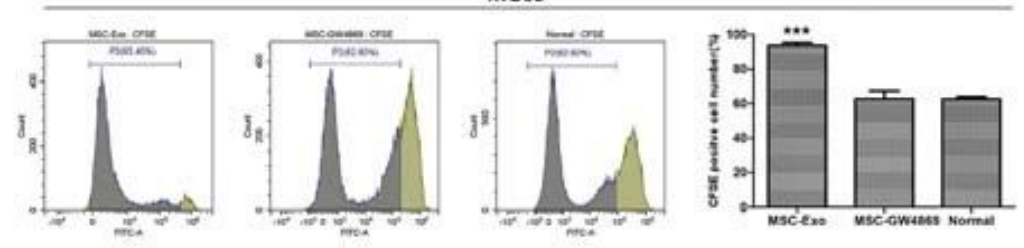

E

C
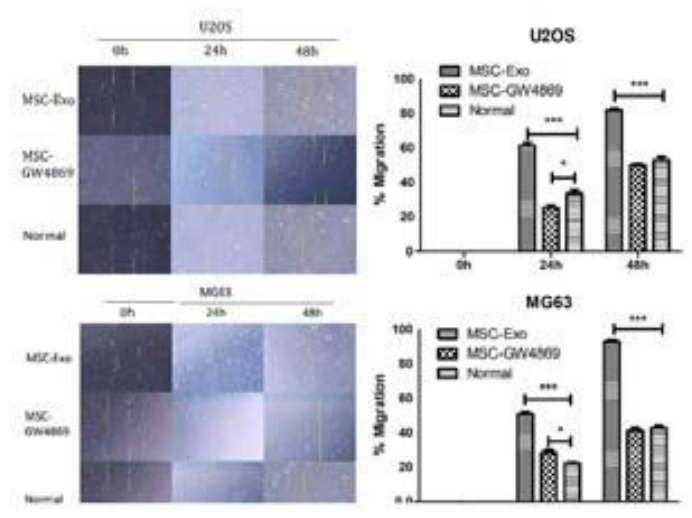

D
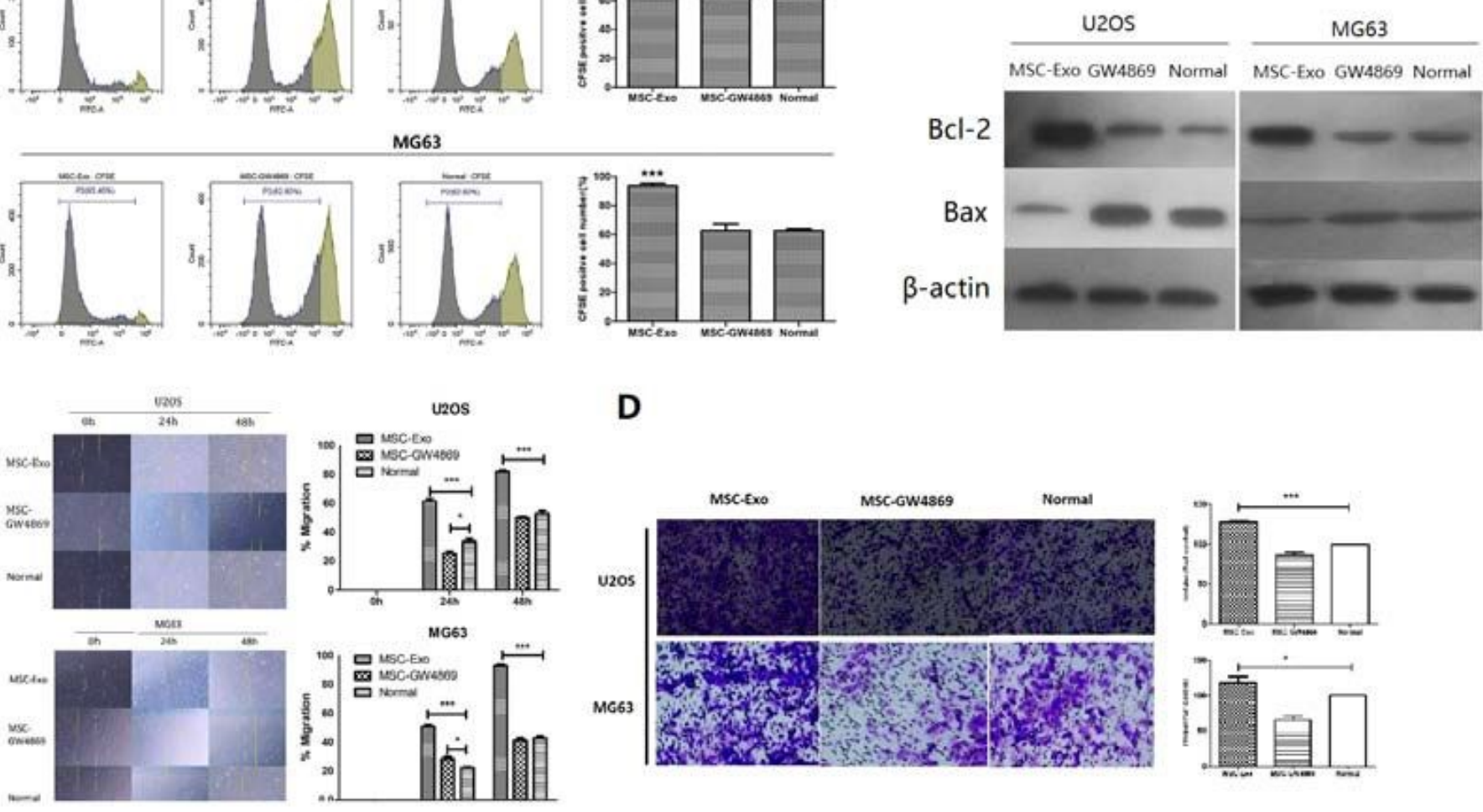

\section{Figure 3}

MSC-derived exosomes promote OS cells proliferation and invasion in vitro. (A)U2OS and MG63 were respectively co-cultured with MSC-derived exosomes and MSC- GW4869 for 24, 48 and 72 hours and then subjected to CCK-8 analyses. Compare with normal group (untreated group), the proliferation rates of both U2OS and MG63 cells were higher after MSC-derived exosomes treatment at 72 hours $(P<0.05)$, whereas there were no significant difference between MSC-GW4869 group and normal group ( $P>0.05)$. (B)From CFSE fluorescence labeling system, the proportions of U2OS and MG63 proliferation in normal group were separately $(65.72 \pm 1.33,62.6 \pm 1.41) \%$, in MSC-Exo group were $(89.64 \pm 2.14,93.40 \pm 1.75) \%$, in MSC-GW4869 group were $(54.95 \pm 4.39,62.8 \pm 4.75) \%$. There were statistical differences between MSCExo group and normal group $(\mathrm{P}<0.001)$, while there were no difference between MSC-GW4869 group and 
normal group (( $P>0.05)$. (C)Scratch wound healing assay test of interfering MSC-derived exosomes group, interfering MSC-GW4869 group and normal group at 24 and 48 hours. The wound healing assay demonstrated a stronger migration ability of U2OS and MG63 in interfering MSC-derived exosomes group. Compared with the normal group and interfering MSC-GW4869 group, there were significant differences in percentage of wound closed at 24 and 48hours $(P<0.001)$,a weaker migration ability of cells in MSC-GW4869 group and normal group.(D)After 24 hours the number of U2OS and MG63 migrated to the lower chamber of the $8 \mu \mathrm{m}$ pore-sized membrane were analyzed by taking photos and counting the number of cells per visual field. Compared with MSC-GW4869 groups and normal groups, the number of U2OS and MG63 were significantly increased 1.283- and 1.187-fold, respectively. $(P<0.05)$. (E)The protein levels of Bcl-2 and Bax in U2OS and MG63 cells of various groups which were evaluated by Western blot. Quantitative data from three independent experiments are shown as the mean $\pm S D$ (error bars). ${ }^{*} P<0.05,{ }^{* *} \mathrm{P}<0.001$. (Student's t-test)

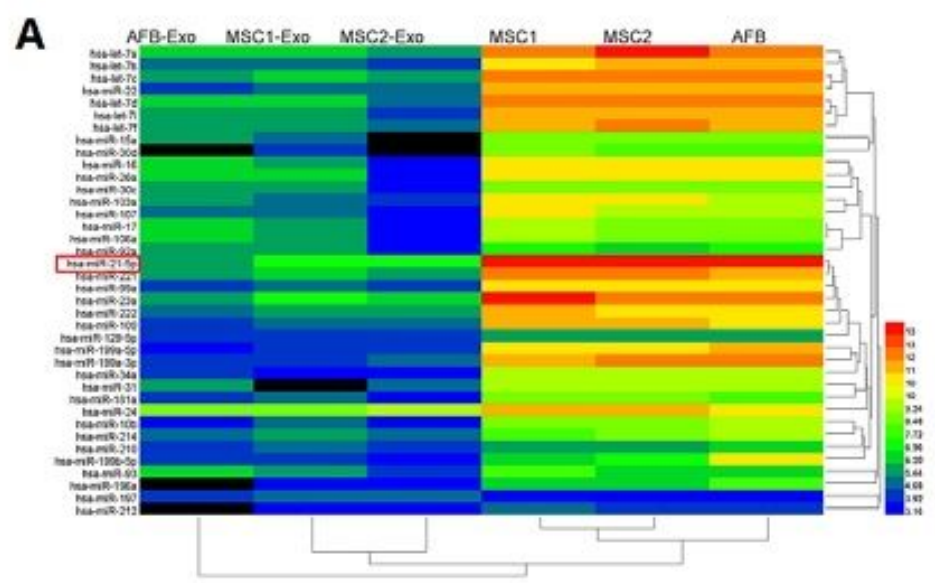

B

PIK3R1 (chr5:67594290-67594296)

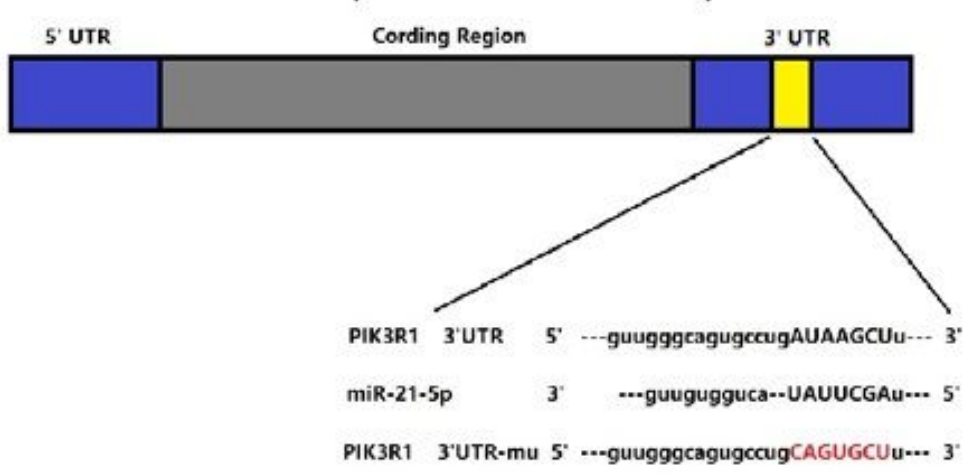

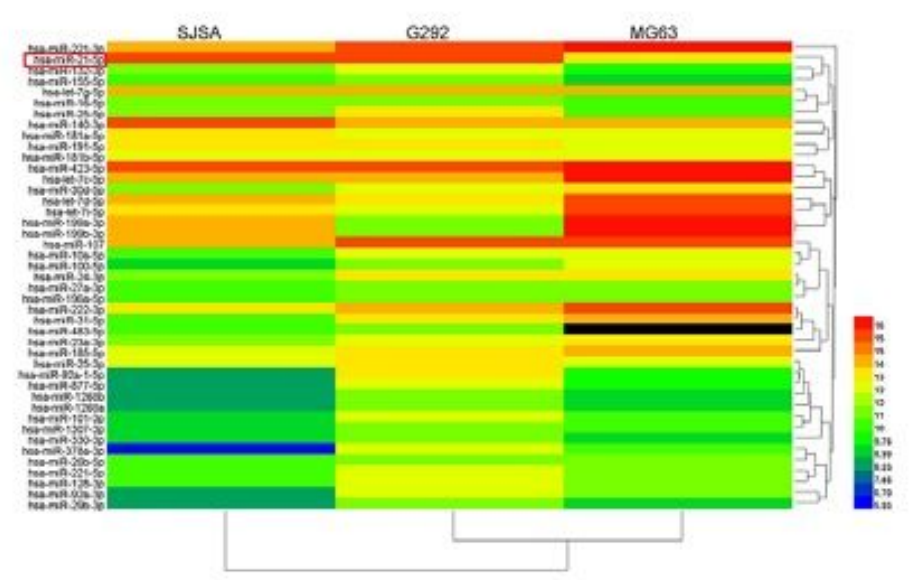

C

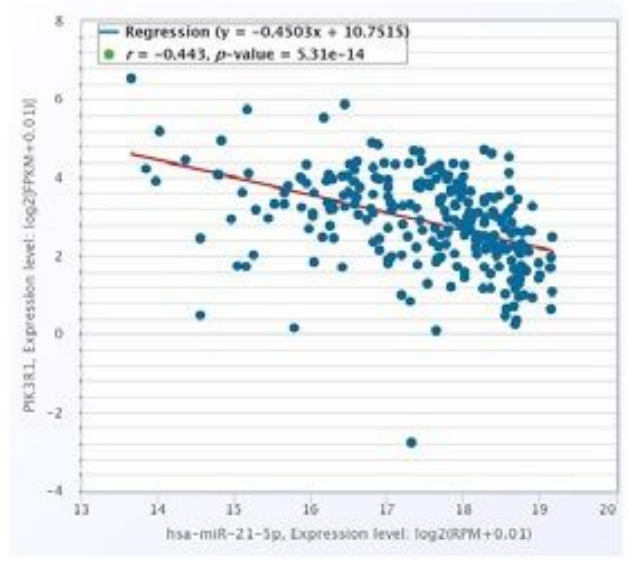

\section{Figure 4}

miRNA expression in human MSC and OS cells. (A) Microarray data obtained from the Gene Expression Omnibus database (Accession No. GSE58027, GSE89930). Heat map shows significant up expression of miR-21-5p in MSCs, MSC-Exo and OS. (blue represents low expression, red represents high expression and black indicates no significant change in gene expression. A color change from blue to red indicates 
upregulation and a color change from red to blue indicates downregulation). (B) Schematic diagram of the miR-21-5p binding site on PIK3R1, bioinformatics analysis using miranda, target scan, encori and pictar indicated that the 3'UTR of PIK3R1 mRNA contains a complementary site for the seed region of miR-21-5p. (C) Pan-Cancer analysis found there was highly negative correlation between miR-21-5p and PIK3R1 in sarcoma.

A
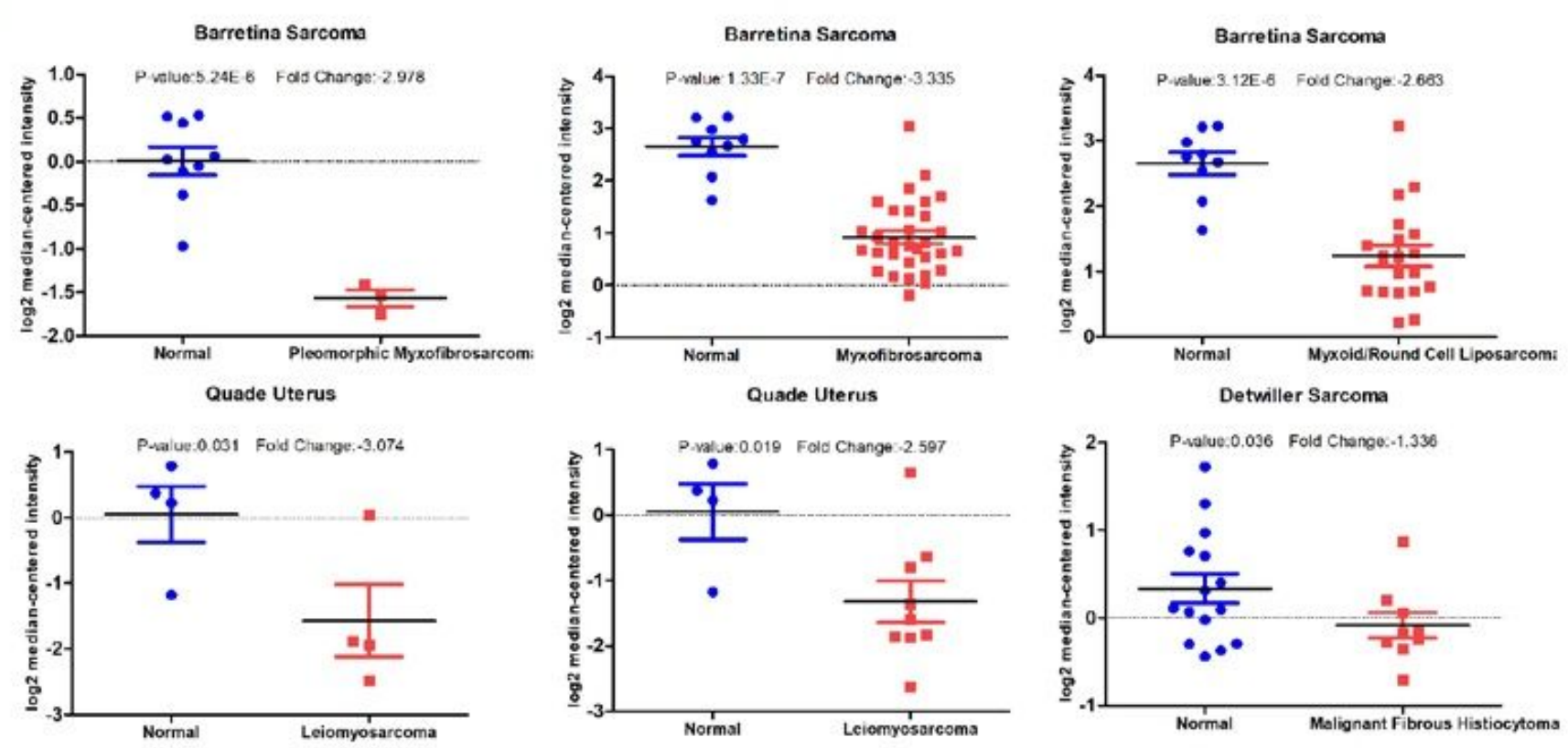

B

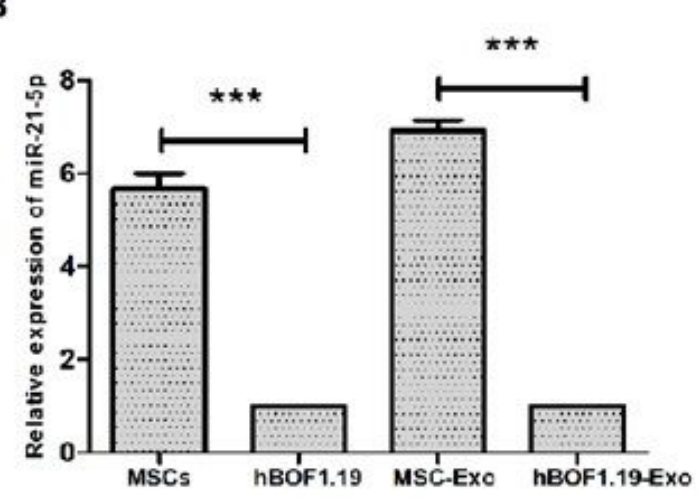

C

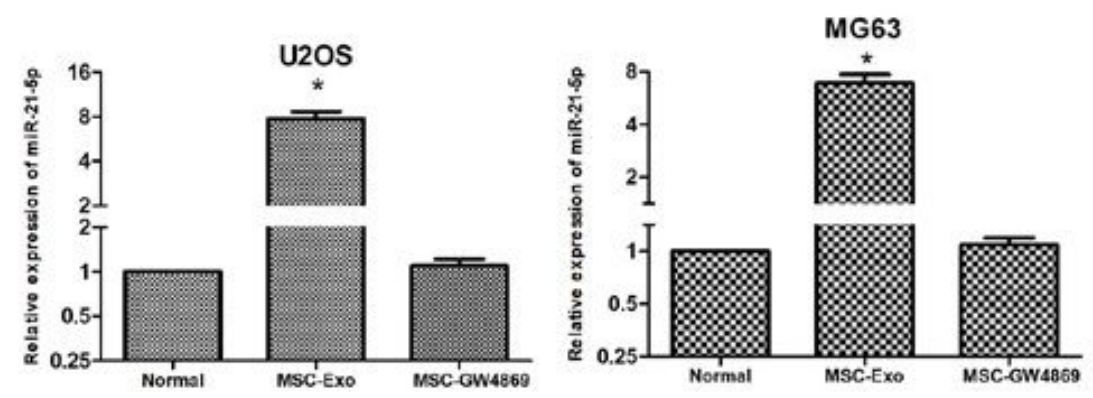

\section{Figure 5}

The expression of PIK3R1 are downregulated in human sarcomas and the expression of miR囚21-5p are upregulated in MSCs, MSC-derived exosomes and OS cells. (A) Analysis of PIK3R1gene expressions in different subtypes of sarcomas using the Oncomine database. Box plot derived from gene expression data in the Oncomine database comparing the expressions of PIK3R1 between normal tissues and cancer tissues in different subtypes of sarcomas, pleomorphic myxofibrosarcoma, myxofibrosarcoma, myxoid/round cell liposarcoma, leiomyosarcoma, and malignant fibrous histiocytoma.(B) The expression of miR囚21-5p in MSCs, hFOB1.19 and their purified exosomes examined by RT-qPCR. (C) The expression of miR-21-5p in U2OS and MG63 transfected with MSC-derived exosomes and MGC-GW8469 for 48 hours 
examined by RT-qPCR. Quantitative data from three independent experiments are shown as the mean \pm $\mathrm{SD}$ (error bars). ${ }^{*} \mathrm{P}<0.05, * * * \mathrm{P}<0.001$ (Student's t-test).

A

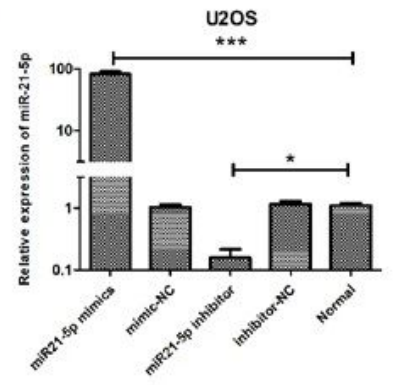

B

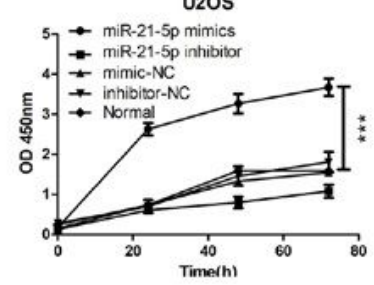

C
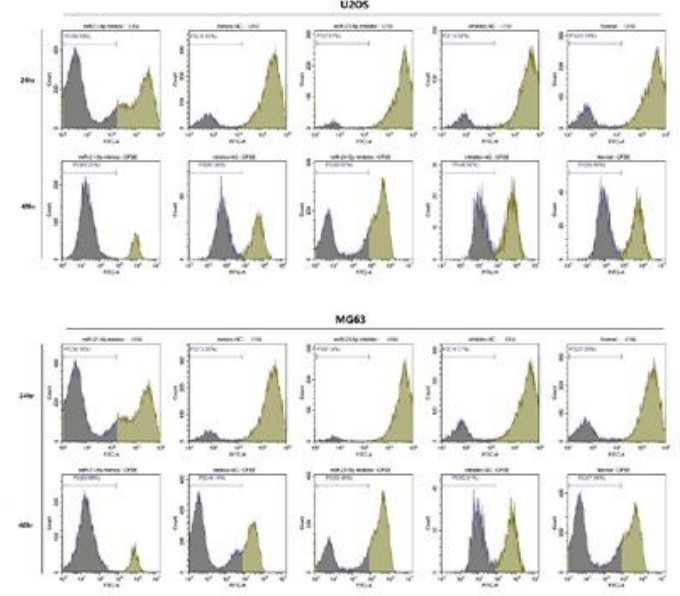

D
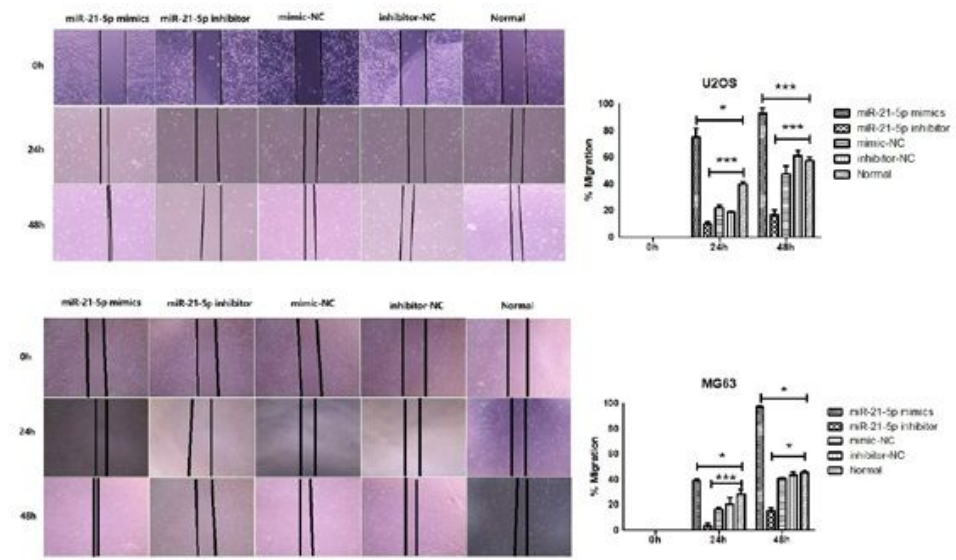
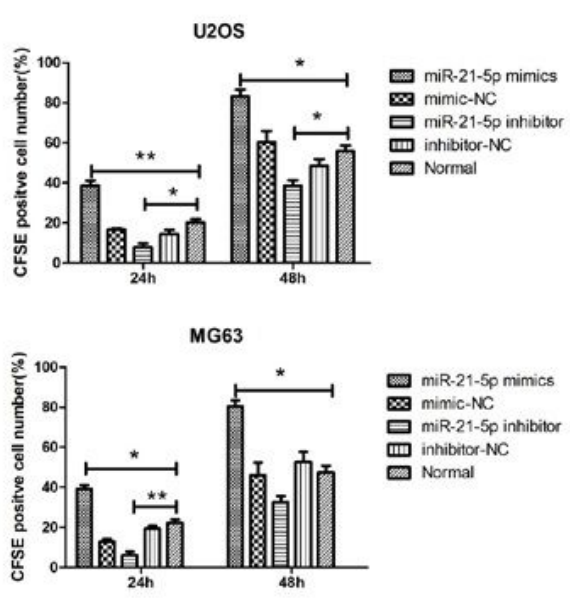

miR-21-5p mimics

mimic-NC

miR-21-5p inhibitor

四 inhibitor-NC

Normal

\section{Figure 6}

Exosomal miR-21-5p derived from MSCs promotes OS cells proliferation and invasion.(A) Expression of miR-21-5p in cultured U2OS and MG63 cells treated with different exosomes derived from different kinds of MSCs (MSCs treated with synthesized miR-21-5p mimic, miR-21-5p inhibitor, mimic-NC, inhibitor-NC, 
and without any treatment). (B) U2OS and MG63 cells proliferation following miR囚21-5p mimic treatment and miR-21-5p inhibitor examined by CCK-8 assay. (C) CFSE fluorescence labeling system further evaluate U2OS and MG63 cells proliferation in different group. (D) Scratch wound healing assay were further conducted to evaluate the effect of exosomal miR-21-5p on the invasive ability of U2OS and MG63.Quantitative data from three independent experiments are shown as the mean \pm SD (error bars). *P $<0.05,{ }^{* *} \mathrm{P}<0.001$. (Student's t-test), miR,microRNA; NC, negative control.

A

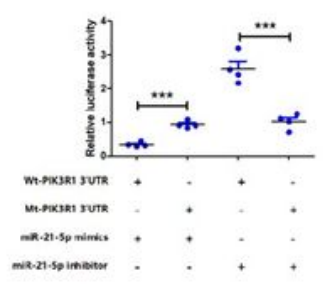

B u20s
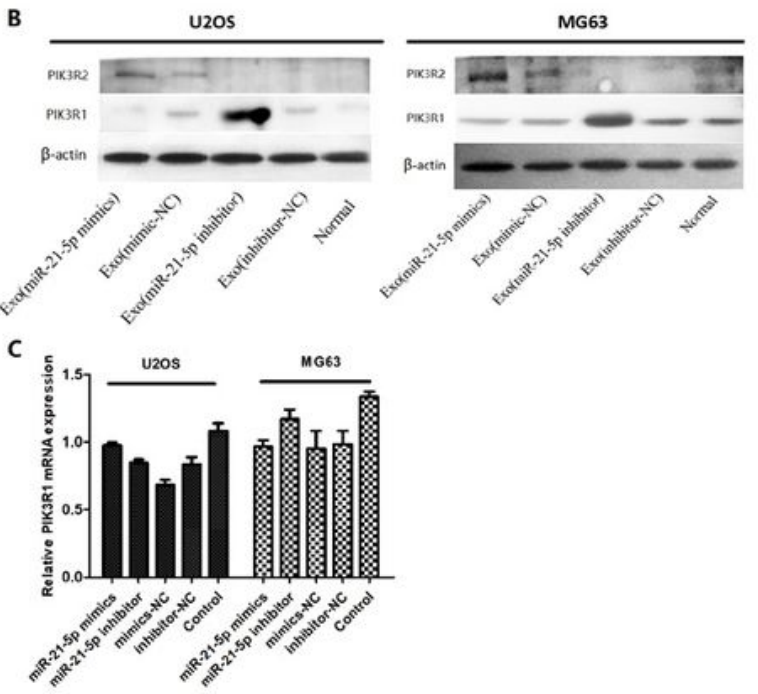

D
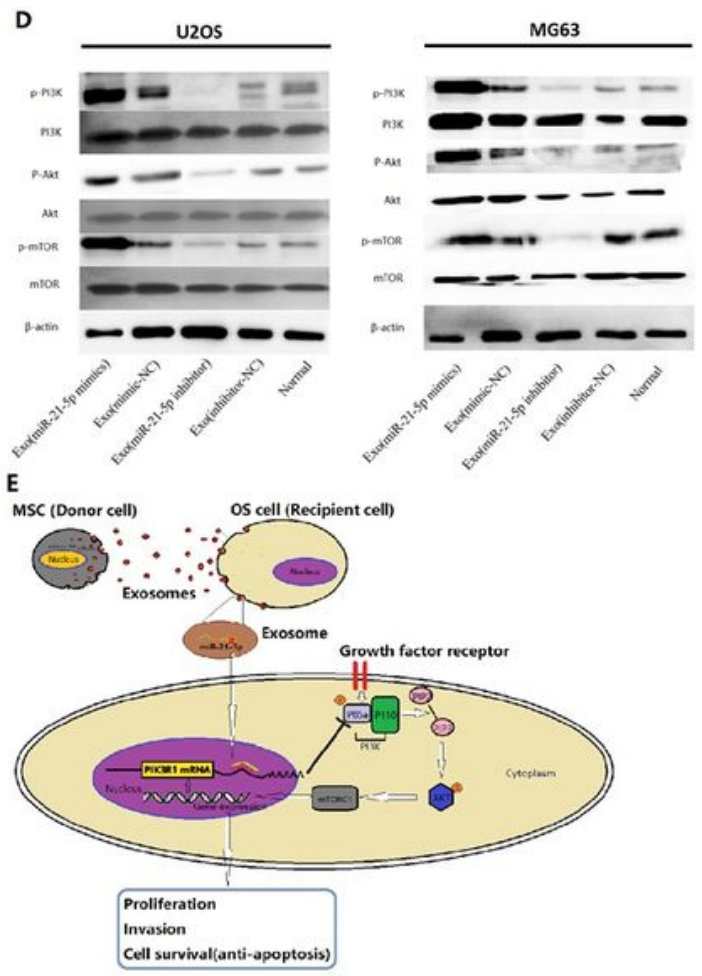

Figure 7 
Exosomal miR-21-5p negatively regulated PIK3R1 and then activated the PI3K/AKT/mTOR signal pathway.(A)Luciferase reporter assays were conducted to validate the interaction between miR-21-5p and the 3'UTR of PIK3R1 ( $n=4$ independent experiments, one-way ANOVA).(B)The western blot assay demonstrated that the protein expression level of PIK3R1 was reduced by miR-21-5p overexpression of MSC-derived exosomes inU2OS and MG63 cells. $\beta$-actin was used as an internal control.(C)T RT-PCR showed the expression level of PIK3R1 have no statistical difference between miR-21-5p inhibitor groups and miR-21-5p mimics groups. (D) Western blot assay was implemented to measure the expression levels of $\mathrm{p}$ - or not PI3K, Akt and mTOR in U2OS and MG-63 after treatment vs normal group. All experiments were implemented in triplicate, ${ }^{*} \mathrm{P}<0.05,{ }^{*} * \mathrm{P}<0.001$. $\mathrm{p}$, phosphorylated; $w \mathrm{t}$, wild-type; $\mathrm{mt}$, mutant; $\mathrm{NC}$, negative control.(E) Schematic of the action of exosomal miR-21-5p derived from human bone marrow MSCs regulating OS cells progression. Human bone marrow MSCs transmit miR-21-5p to OS cells through exosomes, exosomal miR-21-5p target PIK3R1 gene of OS cells, then activating PI3K/Akt/mTOR signal pathway and leading to OS proliferation, invasion and anti-apoptosis. 